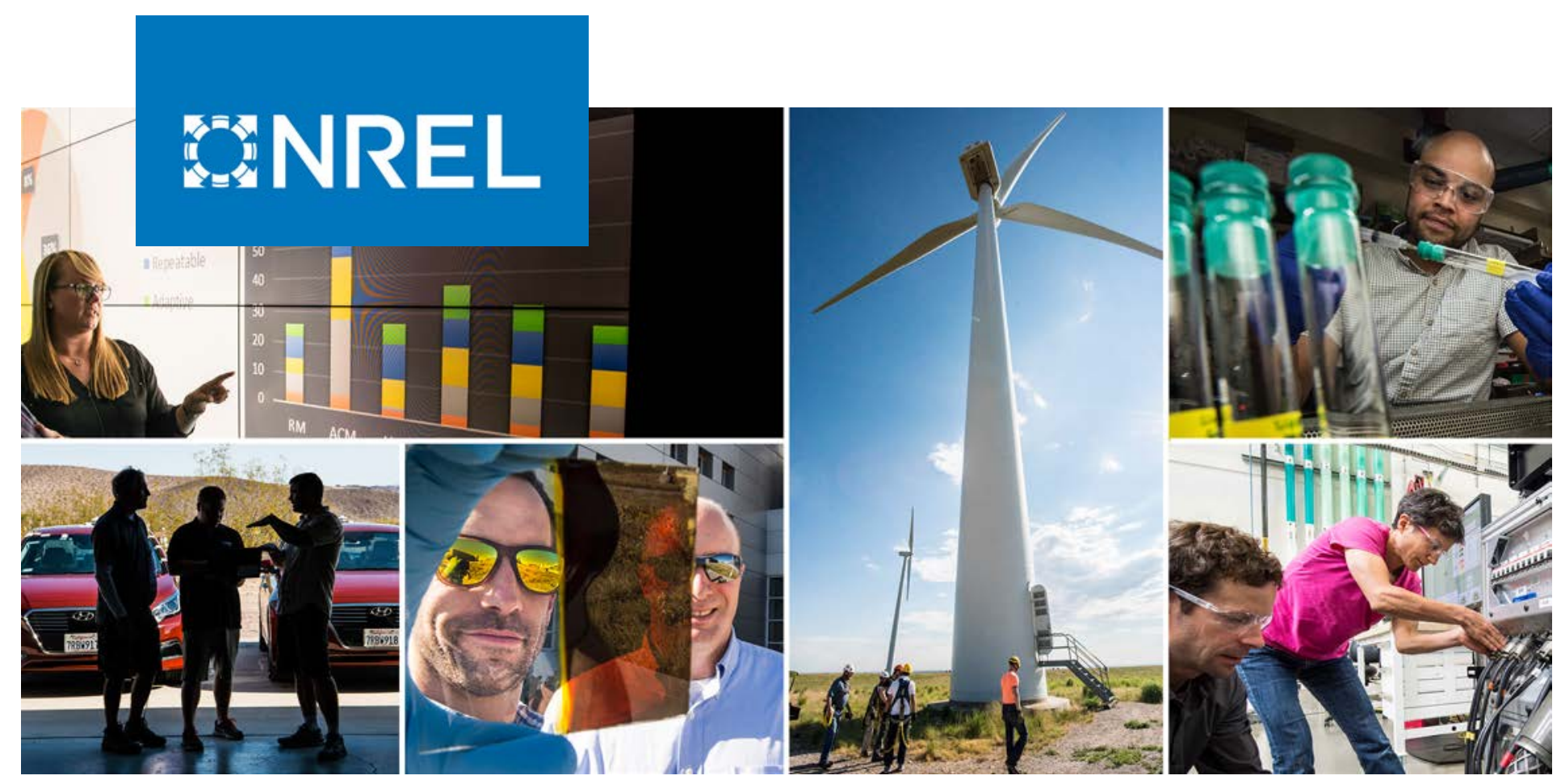

\title{
Ex Situ Catalytic Fast Pyrolysis of Lignocellulosic Biomass to Hydrocarbon Fuels: 2019 State of Technology and Future Research
}

Abhijit Dutta, ${ }^{1}$ Kristiina lisa, ${ }^{1}$ Michael Talmadge, ${ }^{1}$ Calvin Mukarakate, ${ }^{1}$ Michael Griffin, ${ }^{1}$ Eric Tan, ${ }^{1}$ Nolan Wilson, ${ }^{1}$ Matt Yung, ${ }^{1}$ Mark Nimlos, ${ }^{1}$ Joshua Schaidle, ${ }^{1}$ Huamin Wang, ${ }^{2}$ Michael Thorson, ${ }^{2}$ Damon Hartley, ${ }^{3}$ Jordan Klinger, ${ }^{3}$ and $\mathrm{Hao} \mathrm{Cai}^{4}$

1 National Renewable Energy Laboratory

2 Pacific Northwest National Laboratory

3 Idaho National Laboratory

4 Argonne National Laboratory

NREL is a national laboratory of the U.S. Department of Energy Office of Energy Efficiency \& Renewable Energy

Operated by the Alliance for Sustainable Energy, LLC

This report is available at no cost from the National Renewable Energy Laboratory (NREL) at www.nrel.gov/publications.
Technical Report

NREL/TP-5100-76269

March 2020 


\title{
GNREL
}

\section{Ex Situ Catalytic Fast Pyrolysis of Lignocellulosic Biomass to Hydrocarbon Fuels: 2019 State of Technology and Future Research}

\author{
Abhijit Dutta, ${ }^{1}$ Kristiina lisa, ${ }^{1}$ Michael Talmadge, ${ }^{1}$ \\ Calvin Mukarakate, ${ }^{1}$ Michael Griffin, ${ }^{1}$ Eric Tan, ${ }^{1}$ \\ Nolan Wilson, ${ }^{1}$ Matt Yung, ${ }^{1}$ Mark Nimlos, ${ }^{1}$ \\ Joshua Schaidle, ${ }^{1}$ Huamin Wang, ${ }^{2}$ Michael Thorson, ${ }^{2}$ \\ Damon Hartley, ${ }^{3}$ Jordan Klinger, ${ }^{3}$ and $\mathrm{Hao} \mathrm{Cai}^{4}$
}

1 National Renewable Energy Laboratory

2 Pacific Northwest National Laboratory

3 Idaho National Laboratory

4 Argonne National Laboratory

\section{Suggested Citation}

Dutta, Abhijit, Kristiina lisa, Michael Talmadge, Calvin Mukarakate, Michael Griffin, Eric Tan, Nolan Wilson, et al. 2020. Ex Situ Catalytic Fast Pyrolysis of Lignocellulosic Biomass to Hydrocarbon Fuels: 2019 State of Technology and Future Research. Golden, CO: National Renewable Energy Laboratory. NREL/TP-5100-76269. https://www.nrel.gov/docs/fy20osti/76269.pdf.

NREL is a national laboratory of the U.S. Department of Energy Office of Energy Efficiency \& Renewable Energy Operated by the Alliance for Sustainable Energy, LLC

This report is available at no cost from the National Renewable Energy Laboratory (NREL) at www.nrel.gov/publications.

Contract No. DE-AC36-08GO28308
Technical Report NREL/TP-5100-76269 March 2020

National Renewable Energy Laboratory 15013 Denver West Parkway Golden, CO 80401 303-275-3000 • www.nrel.gov 


\section{NOTICE}

This work was authored in part by the National Renewable Energy Laboratory, operated by Alliance for Sustainable Energy, LLC, for the U.S. Department of Energy (DOE) under Contract No. DE-AC36-08GO28308. Funding provided by U.S. Department of Energy Office of Energy Efficiency and Renewable Energy Bioenergy Technologies Office. The views expressed herein do not necessarily represent the views of the DOE or the U.S. Government.

This report is available at no cost from the National Renewable Energy Laboratory (NREL) at www.nrel.gov/publications.

U.S. Department of Energy (DOE) reports produced after 1991 and a growing number of pre-1991 documents are available free via www.OSTI.gov.

Cover Photos by Dennis Schroeder: (clockwise, left to right) NREL 51934, NREL 45897, NREL 42160, NREL 45891, NREL 48097, NREL 46526.

NREL prints on paper that contains recycled content. 


\section{Acknowledgments}

The authors wish to thank the following researchers for their contributions to this work: Richard French, Kellene Orton, Scott Palmer, Braden Peterson, Kim Magrini, Joseph Roback, Kylee Harris, Dan Ruddy, Frederick Baddour, Kurt Van Allsburg, Daniel Carpenter, and Zia Abdullah from the National Renewable Energy Laboratory (NREL), Daniel M. Santosa from Pacific Northwest National Laboratory (PNNL), and Bruce Adkins from the Oak Ridge National Laboratory. We appreciate communications support from Kathy Cisar and Liz Breazeale from NREL. We thank Mike Watson and Luke Tuxworth from Johnson Matthey for their cooperative work with NREL on catalyst research (under a cooperative research and development agreement).

This effort is supported by many individuals across multiple national laboratories. The authors apologize in advance for inadvertent omissions in acknowledging any contributors to this project. 


\section{List of Acronyms}

AAEM

ANL

Btu

CFP

Co-HP

DCFROR

FCI

FY

GGE

GHG

HGF

INL

ISBL

LCA

LHV

MEK

MFSP

NREL

PNNL

MM

PSA

SCSA

SOT

TCI

TDC

TEA

TIC

TPEC

WHSV alkali and alkaline earth metal

Argonne National Laboratory

British thermal unit

catalytic fast pyrolysis

co-hydroprocessing

discounted cash flow rate of return

fixed capital investment

fiscal year

gallon gasoline equivalent

greenhouse gas

hot gas filter

Idaho National Laboratory

inside battery limits

life-cycle analysis

lower heating value

methyl-ethyl-ketone

minimum fuel selling price

National Renewable Energy Laboratory

Pacific Northwest National Laboratory

million

pressure swing adsorption

supply chain sustainability analysis

state of technology

total capital investment

total direct cost

techno-economic analysis

total installed cost

total purchased equipment cost

weight hourly space velocity 


\section{Executive Summary}

\section{Structure of This Report}

The goal of this executive summary is to present recent research outcomes for the biomass ex situ catalytic fast pyrolysis (CFP) conversion pathway in the context of techno-economic analysis (TEA), and provide a clear understanding of our rationale behind research decisions, without the reader having to search for details within the report. Key summary tables are also included at the end of this executive summary. Quantitative details are presented in the main body of this report with focus on the 2019 State of Technology (SOT) and a 2020 projection case with co-hydroprocessing (co-HP); background information is provided for anyone familiar with biomass CFP to comprehend the text without having to refer to additional material. Interested readers, and those unfamiliar with biomass CFP, are provided with more detailed references for additional background. Detailed cost breakdown information is presented in the Appendix.

\section{Background Information}

This report documents the progress in research funded by the U.S. Department of Energy's Office of Energy Efficiency and Renewable Energy Bioenergy Technologies Office for the conversion of biomass to infrastructure-compatible liquid hydrocarbon fuels via CFP; the focus is on research learnings since a 2018 SOT publication. ${ }^{i}$

In the ex situ CFP pathway, biomass undergoes rapid deconstruction in a fast pyrolysis reactor at approximately $500^{\circ} \mathrm{C}\left(932^{\circ} \mathrm{F}\right)$, followed by the separation of produced solids (char and mineral matter) from vapors (including permanent gases); the vapors are then sent to an ex situ catalytic reactor for upgrading. Upgrading involves deoxygenation, hydrogenation, and carbon-carbon coupling, and this renders the vapors significantly less reactive and more amenable to further processing after condensation (the condensation product is known as CFP oil). Solids removal prior to the ex situ upgrading step provides an advantage with respect to catalyst stability and choices (relative to in situ upgrading where the catalyst mixes with biomass-derived solids within the fast pyrolysis reactor); catalyst choices can be further broadened to include supported noble metals in fixed bed systems. The effectiveness of this ex situ vapor upgrading step for CFP oil quality improvement has been verified, with experiments proving that single-step hydrotreating can deoxygenate the liquid product to less than $1 \mathrm{wt} \%$ oxygen. Catalyst stability during hydrotreating of raw fast pyrolysis bio-oil is a major challenge; multiple hydrotreating steps are required unless the catalytic vapor upgrading step is included. Initial research efforts associated with this project (beginning in 2014) were focused on zeolite catalysts in a fluidized reactor system; this was detailed in a 2015 design report. ${ }^{\text {ii }}$ The 2018 SOT report documented significant liquid-range product yield improvements in 2017 using a $\mathrm{Pt} / \mathrm{TiO}_{2}$ catalyst that led to the choice of a fixed bed ex situ configuration for further research improvements beyond 2017. The significant modeled minimum fuel selling price (MFSP) reduction in 2018 was because of a reduced $\mathrm{Pt}$ loading of $0.5 \%$ on the $\mathrm{Pt} / \mathrm{TiO}_{2}$ catalyst, compared to $2 \%$ in 2017 , and increased ratio of online to regeneration time (reduction from $2: 5$ to $2: 3$ ).

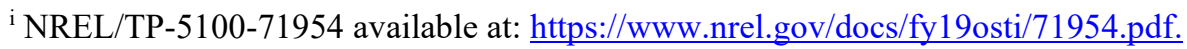

ii NREL/TP-5100-62455, PNNL-23823 available at: https://www.nrel.gov/docs/fy15osti/62455.pdf.
} 


\section{Research and Development Since the 2018 SOT Report}

\section{Carbon Balance Closure}

Through 2018, there were uncertainties regarding the product distribution for the CFP step because experimentally measured carbon balance closures were $88 \%$ and $87 \%$ in the 2017 and 2018 SOT experiments, respectively. A decision was made to tackle this uncertainty by deploying additional analytical equipment (a Polyarc-GC-MS system) in 2019, which helped achieve a nearly $100 \%$ carbon balance closure. Our previous approach was to prorate the missing carbon among the different phases (solid char, condensable liquids, and permanent gases), with some conservatism toward the carbon allocated to the liquid phase fuel-precursor products because fuel yield improvements significantly benefit the economics of the process. The 2018 SOT MFSP was initially evaluated at $\$ 3.50$ /gallon gasoline equivalent (GGE), with sensitivity analysis capturing the effect of lower yields of liquid fuel. The new measurements in 2019 showed that the majority of the missing carbon was in light oxygenated species. This quantification had a significant impact on the economics of the conceptual process design because hydrotreating those light oxygenates would result in gaseous hydrocarbons that do not count towards our desired liquid-range hydrocarbon fuel products. The 2018 SOT was revised after discounting the organic phase products that do not produce liquid hydrocarbons; the carbon efficiency towards the organic intermediate used for producing liquid hydrocarbon fuels was revised downward from $44.7 \%$ to $40.4 \%$, resulting in an upward revision of the 2018 SOT MFSP from $\$ 3.50 / \mathrm{GGE}$ to $\$ 3.80 / \mathrm{GGE}$. This revision did not include any efforts towards the recovery and utilization of the newly quantified light oxygenates that account for $>10 \%$ of the biomass carbon.

\section{Recovery of Light Oxygenates}

The additional quantification of product species required research towards the effective recovery and utilization of the light oxygenates to benefit the economics of the process. The results showed that there is significant selectivity toward three specific compounds (among all the light oxygenates and some light hydrocarbons): (a) acetaldehyde, (b) acetone, and (c) 2-butanone (or methyl-ethyl-ketone [MEK]) together accounted for $\sim 90 \%$ of the mass of $\mathrm{C}_{4}$ - species, and $>75 \%$ of the mass after including $\mathrm{C}_{5+}$ species of light compounds. Yields to these three compounds accounted for $>7.5 \%$ of the total initial biomass carbon. The high selectivity offered an option to recover, separate, and sell these oxygenated products into existing markets. The removal of these compounds would also reduce hydrogen demand for the subsequent hydroprocessing step to produce hydrocarbon fuel blendstocks. The TEA model was modified to reflect this approach. The separation strategy was based on an adsorption/desorption cycle to trap the light oxygenates, followed by distillation of the relatively smaller stream of desorbed condensables to recover purified products. Model compound mixtures were used in experiments to measure adsorption/desorption performance; different adsorbents were tested to prove that we indeed have an adsorbent that can capture the desired compounds. Adsorption/desorption experiments on biomass CFP vapors will be performed in the near future to validate the TEA assumptions (TEA assumptions are currently based on model compounds). In addition, there are uncertainties related to effective distillation and purification of acetone and MEK from the desorbed stream containing other compounds. Contingencies were added to the TEA to cover additional costs; as noted, the desorbed stream will have a relatively small flow rate and additional capital and operating costs will likely be covered under our assumed contingency. We will however need to 
fully quantify the desorbed stream to prove our approach of final product purification via distillation. The detailed stream composition will be used in our Aspen Plus model to quantify the potential product purity and recovery; follow-up experiments will be conducted if necessary. While acetone and MEK have existing markets that can absorb/consume the production from multiple biorefineries at the 2,000 dry metric tons per day scale (the basis of the conceptual design models used for the TEA), acetaldehyde does not have a significant market and cannot be sold directly after separation. Thus, acetaldehyde was not considered for near term valorization, although significant research opportunities exist for the conversion of a nearly pure acetaldehyde stream into other marketable products. The sale of these coproducts (acetone and MEK at this point) helped reduce the MFSP for fuel blendstocks by 52 cents/GGE in the 2019 SOT model.

\section{CFP Catalyst Onstream Time}

Another major development was the use of a modified support structure for the $\mathrm{Pt} / \mathrm{TiO}_{2}$ catalyst for CFP experiments in 2019. This catalyst was able to maintain a predetermined performance threshold for 8 hours before requiring regeneration; this is a significant improvement over the 2018 catalyst. The previous 2 -hour onstream time in 2018 was a major concern for commercial implementation of this fixed bed ex situ CFP system. Further improvements are being made to extend the onstream time. The current performance allows a 1:1 ratio of online:regenerating reactors. The enhanced onstream time was achieved despite a decrease in the CFP oil oxygen content compared to the FY18 SOT, from $19 \mathrm{wt} \%$ to $15 \mathrm{wt} \%$ (dry basis). However, this new catalyst resulted in a reduction in the CFP oil carbon yield to $35 \%$ in 2019 compared to the revised 2018 SOT yield of $40 \%$. It is likely that there is scope for optimization of the 2019 catalyst performance via adjustments in operating conditions and space velocities; this is expected to be explored during a 500-hour bench-scale experimental campaign in 2020 .

\section{Use of a Lower-Cost Feedstock}

Another 2019 SOT highlight was the successful use of a lower-quality (and lower-cost) feedstock that incorporated 50\% forest residues with 50\% clean pine (compared to 100\% clean pine used in previous years). This decreased the feedstock cost to $\$ 70 /$ dry US ton vs the 2018 value of $\$ 88 /$ dry US ton. The modeled 2019 SOT MFSP was $\$ 3.33 /$ GGE using the $\$ 70 /$ dry US ton feedstock cost; using the $\$ 88 /$ dry US ton feed cost would have given a modeled MFSP of $\$ 3.63 /$ GGE. Long-term impacts of the additional mineral matter from forest-residues on the ex situ CFP catalyst will be studied as part of the 500-hour run planned in 2020. Additional operations to further clean up mineral matter from forest-residues are being explored at the Idaho National Laboratory (INL). The current 2021-2022 projections tentatively use a $75 \%$ forest residues $/ 25 \%$ clean pine blend with mineral matter content reduced below $1 \%$. The impacts of any additional cleanup on CFP performance will be studied. The final feedstock choice for a 2022 verification will be dictated by the performance of the current $50 \%$ forest residues $/ 50 \%$ clean pine mix during the 500-hour run.

\section{CFP Oil Co-Hydroprocessing Strategy}

Past SOTs have quantified standalone single-stage hydrotreating performance of CFP oils. Cohydroprocessing the CFP oil in petroleum refineries is also an option being considered. This approach can facilitate significant cost-savings (this is in addition to recovering coproducts and using lower cost feedstocks discussed above). CFP-oil can be co-hydroprocessed with streams at petroleum refineries by utilizing existing hydroprocessing capital and hydrogen infrastructure 
and avoiding the investment in these facilities at a smaller scale (with higher unit processing costs) at the biorefinery. However, streams such as CFP oil with high oxygen content of 15-20\% have never been introduced into mainstream petroleum refining processes. The key challenges for a co-hydroprocessing approach include: (1) proving the feasibility of the coprocessing with appropriately compatible petroleum refinery units/streams to achieve desired product quality (including reduction in oxygen content and associated reactivity), (2) identifying potential disruptive impacts on petroleum refinery operations (e.g., operational upsets such as plugging, corrosion, catalyst life, fuel quality) and developing/proving ways to mitigate them, and (3) engaging petroleum refining industry experts to allow buy-in of this approach through diligent joint investigation. Before investigating this approach from a TEA perspective, preliminary cohydrotreating experimental data generated at PNNL under the Strategies for Co-Processing in Refineries (SCR) project ${ }^{\mathrm{iii}}$ and NREL were used to understand whether this approach would be feasible. Experiments with a straight-run diesel fraction and 5-20 wt\% CFP oil (from two different catalysts) showed effective deoxygenation of the product, maintenance of carbon efficiencies attributed to the CFP oil portion (compared to standalone hydrotreating of CFP oil), and similarities in fuel properties obtained from hydrotreating a straight run diesel stream (distillation curves with small parallel shifts were observed for one experimental data set and acceptable cetane numbers of greater than 40 were measured for a different experiment). The TEA approach for co-hydroprocessing used hydrogen consumption, the anticipated primary cost driver for this step at a petroleum refinery, as the key variable. Refinery costs were estimated using hydrogen consumption as the basis variable, with information derived from Gary et al. ${ }^{\text {iv }}$ The TEA results showed a reduction in the modeled MFSP from \$3.33/GGE for the 2019 SOT to $\$ 3.09 /$ GGE in 2020 (option case presented in this report), with the cost savings attributed entirely to the switch from standalone hydroprocessing to co-hydroprocessing. Life cycle assessment for this scenario (presented as a 2020 option in this report) showed a greater than $50 \%$ greenhouse gas (GHG) reduction (compared to petroleum derived gasoline) for the entire production chain from biomass to hydrocarbon fuels (after also accounting for the light-oxygenated coproducts mentioned previously). The hydrogen estimates will be updated in the future; lower hydrogen consumption can allow further GHG reduction. The other area for GHG reduction is during feedstock preprocessing, which will also be considered in an integrated manner as part of life cycle impacts for the entire supply chain.

\section{Research Plans}

Current research plans include a potential verification of key parts of this technology in 2022. This verification is designed to facilitate future scale-up by interested industrial entities. A 500hour bench scale experimental campaign for CFP is expected to be conducted in 2020 to allow the selection of various components to be used during the verification. Corresponding hydroprocessing of the CFP oil, with 500-hour or longer run-times, is also planned. The TEA projections for 2020 presented below include two options for achieving an MFSP of approximately $\$ 3.09 / \mathrm{GGE}$. One of the options includes co-hydroprocessing with petroleum refinery streams, while the other involves an increase in fuel yields via the optimization of

\footnotetext{
iii Baldwin et al. Bioenergy Technologies Office. Peer Review presentation March 2019. Available at: https:/www.energy.gov/sites/prod/files/2019/04/f61/Strategies $\% 20$ for $\% 20 \mathrm{Co}-$ Processing\%20in\%20Refineries\%20\%28SCR\%29 NL0032422.pdf.

iv Gary,J.H; Handwerk,G.E;Kaiser,M.J. Petroleum Refining: Technology and Economics, $5^{\text {th }}$ Edition. CRC Press.
} 
process conditions. Further cost reduction towards achieving $\$ 3 /$ GGE will be explored based on the 2020 experimental results.

\section{Summary of Key Results}

The following figures and tables summarize key results from experiments and related analysis described above. The tables below include information about the updated 2018 SOT and the 2019 SOT, and two options for a 2020 projection of \$3.09/GGE. Additional information, including information for 2014-2017 SOTs and projections for 2021-2022 are presented in the Appendix. Note that the 2021-2022 projections presented are preliminary and will be updated based on the outcome and learnings from the 500-hour run and a decision about our choice of one of the two options presented for 2020. An option with projections based on cohydroprocessing with additional light oxygenated coproducts recovery improvements was used as the placeholder for 2021-2022; additional benefits may result from using a lower ash feedstock at a comparable cost and a modest increase in fuel blendstock yield. The coproduct recovery improvements can be shown outside the scale-up efforts for the 2022 verification, thus minimizing additional changes to that setup. It should be noted that other research improvements are also expected to continue at the bench scale during the 2021-2022 time frame and some of those aligned improvements may be leveraged to scientifically justify additional cost reduction possible at the verification scale.

\section{SOT}

The primary uncertainty for the 2019 SOT assessment lies in our assumption regarding coproduct recovery and purification, since mass and carbon balances are well quantified at this point. The base case MFSP reported for the 2019 SOT is $\$ 3.33 /$ GGE. The following variations were considered because of our current use of model compound information in the TEA:

- The acetone and MEK product prices assumed for the TEA were derived from 5-year averages. A $90 \%$ of a 5 -year average price was used for both acetone ${ }^{\mathrm{v}}$ and MEK, ${ }^{\mathrm{vi}}$ with respective coproduct values of $39.8 \not / \mathrm{lb}$ and $69.4 \phi / 1 \mathrm{~b}$ respectively; the $90 \%$ factor was used in this particular instance to cover uncertainties related to product purity and associated value. Decreasing the coproduct values further to $70 \%$ (which may happen in the event that more significant impurities are not mitigated by additional purification steps at the biorefinery) increased the MFSP to $\$ 3.44 / \mathrm{GGE}$;

- Reducing the equipment cost contingency by $\$ 3.5$ million dropped the MFSP to $\$ 3.28 / \mathrm{GGE}$, while adding $\$ 3.0$ million to the equipment cost increased the MFSP to $\$ 3.37 / \mathrm{GGE}$; and

- Dropping acetone and MEK recovery by $20 \%$ increased the MFSP to $\$ 3.43 / \mathrm{GGE}$.

Based on these uncertainties, we report a modeled MFSP range of $\$ 3.28 / \mathrm{GGE}$ to $\$ 3.44 / \mathrm{GGE}$ associated with the base case value $\$ 3.33 / \mathrm{GGE}$.

\footnotetext{
v Pampell, M.; Spyra, T. Acetone. Chemical Economics Handbook, IHS Markit. August 15, 2018.

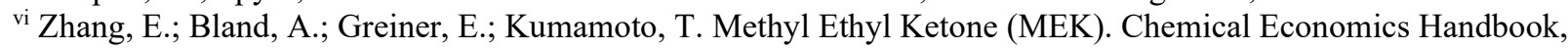
IHS Markit. August 31, 2018.
} 


\section{Sensitivity Analysis}

Sensitivity analysis for key uncertainties for the 2020 co-hydroprocessing case are shown in Figure ES- 2 below. Some of the sensitivity analyses included in the 2018 SOT report $^{\mathrm{i}}$ are still relevant and all those cases are not repeated in this report. The 2020 petroleum refinery coprocessing option is used as a basis for Figure ES- 2 because the current analysis covers some additional uncertainties related to co-hydroprocessing. Note that a CFP oil cost of $\$ 121 /$ barrel gasoline equivalent product is estimated for this 2020 case. The (barrel gasoline equivalent product) unit was used for volume to avoid confusion about the different density of CFP oil intermediate compared to conventional petroleum refinery streams; the product basis in this case is the final hydrocarbon fuel output from the CFP oil.

\section{Summary of Figures and Tables Presented Below}

- Figure ES- 1: Waterfall chart showing previous cost reduction and future projections

- Table ES-1: Key metrics for the updated 2018 SOT, 2019 SOT, and 2020 projections with two options

- Table ES-2: Modeled economic summary for the updated 2018 SOT case

- Table ES-3: Modeled economic summary for the 2019 SOT case

- Table ES- 4: Modeled economic summary for the 2020 projection with a co-hydroprocessing option

- Figure ES- 2: Sensitivity analysis for the 2020 model with co-hydroprocessing

- Table ES- 5: Modeled economic summary for the 2020 projection with a yield increase option.

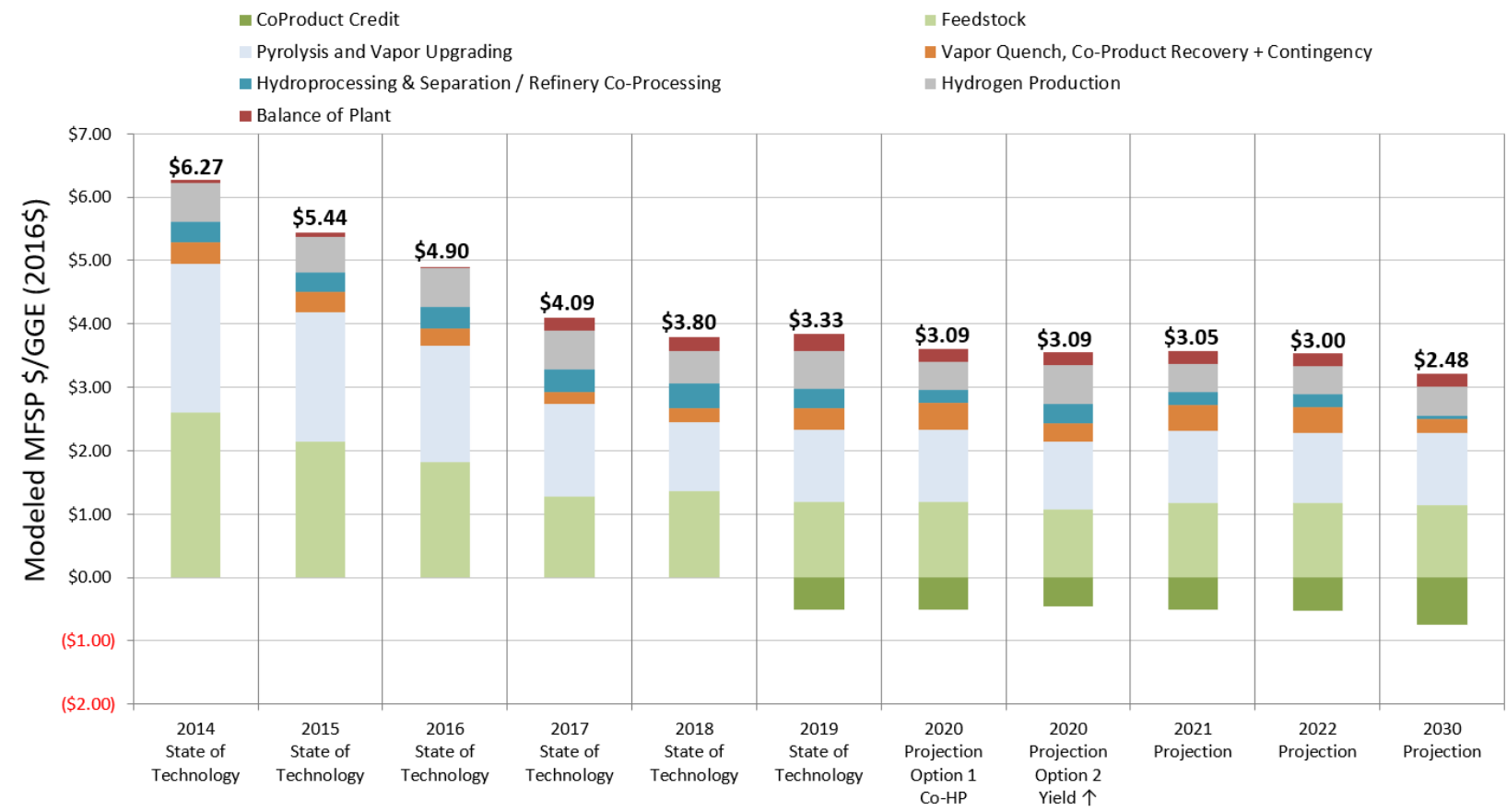

Figure ES-1. Modeled MFSP for 2014-2019 SOTs and 2020-2022 projections. Two options are presented for cost reduction in 2020: (1) coproducts with refinery co-hydroprocessing and (2) coproducts with a higher fuel yield at the biorefinery. 2030 projection is very preliminary based on additional coproducts and CFP yield improvements. 
Table ES-1. Key Metrics for Updated Fiscal Year (FY) 2018 SOT, 2019 SOT, and 2020 Projections

\begin{tabular}{|c|c|c|c|c|}
\hline & $\begin{array}{r}\text { Updated } \\
2018 \text { SOT }^{a} \\
\end{array}$ & $\begin{array}{r}\text { FY } 2019 \\
\text { SOT }\end{array}$ & $\begin{array}{r}F Y 2020 \\
\text { Co-HP }\end{array}$ & $\begin{array}{r}\text { FY } 2020 \\
\text { Yield } \uparrow\end{array}$ \\
\hline \multicolumn{5}{|l|}{ Fast Pyrolysis Intermediate ${ }^{b}$} \\
\hline Gas Species-CO, $\mathrm{CO}_{2}, \mathrm{C}_{1}-\mathrm{C}_{4}$ (wt\% of dry biomass) & 13 & 13 & 13 & 13 \\
\hline Organics (wt\% of dry biomass) & 64 & 64 & 64 & 64 \\
\hline Water (wt $\%$ of dry biomass) & 11 & 11 & 11 & 11 \\
\hline Char (wt\% of dry biomass) & 12 & 12 & 12 & 12 \\
\hline Fixed Bed-Online: Regen Reactors ${ }^{e}$ & $2: 3$ & 2:2 & $2: 2$ & 2:2 \\
\hline Gas, Includes Condensables (wt \% of dry biomass) & 35 & 38 & 38 & 34 \\
\hline Aqueous Phase (wt\% of dry biomass) & 22 & 24 & 24 & 26 \\
\hline Carbon Loss ( $\%$ of C in biomass) & 5 & 4.4 & 4.4 & 4.3 \\
\hline Organic Phase (wt\% of dry biomass) & 28 & 23 & 23 & 26 \\
\hline H/C Molar Ratio & 1.2 & 1.2 & 1.2 & 1.2 \\
\hline Oxygen (wt\% in organic phase) & 18.6 & 15 & 15 & 15 \\
\hline Carbon Efficiency (\%) & 40.4 & $35^{f}$ & $35^{f}$ & $39^{f}$ \\
\hline Solid Losses, Char + Coke (wt $\%$ of dry biomass) & $12+4^{c}$ & $12+2$ & $12+2$ & $12+2$ \\
\hline \multicolumn{5}{|l|}{ Final Fuel Blendstock } \\
\hline Yield (\%, w/w dry biomass) & 21 & 19 & 19 & 21 \\
\hline Hydroprocessing Carbon Efficiency (\%) e & 89 & 93.5 & $93.5 \mathrm{~g}$ & 93.5 \\
\hline Overall Carbon Efficiency to Fuel (\% of C in biomass) & 36 & 33 & 33 & 36 \\
\hline Overall C-Efficiency to Fuel (\% of C in biomass + NG) & 36 & 33 & 33 & 36 \\
\hline Total Product (GGE/dry U.S. ton) & 65 & 59 & 59 & 65 \\
\hline Gasoline-Range Product (gallons/dry U.S. ton) & 33 & 32 & 32 & 35 \\
\hline Diesel-Range Product (gallons/dry U.S. ton) & 31 & 26 & 26 & 29 \\
\hline Gasoline/Diesel-Range Product (\% GGE basis) & $48 / 52$ & $52 / 48$ & $52 / 48$ & $52 / 48$ \\
\hline Oxygen Content in Cumulative Product (wt\%) & 0.5 & 0.5 & 0.5 & 0.5 \\
\hline Acetone + MEK Coproduct Credit ( $\phi / G G E)$ & - & 52 & 52 & 47 \\
\hline Minimum Fuel Selling Price (\$/GGE) & 3.80 & 3.33 & 3.09 & 3.09 \\
\hline \multicolumn{5}{|l|}{ Natural Gas ${ }^{\dagger}$ and Electricity } \\
\hline Natural Gas Energy Input (\% of biomass, LHV basis) & 0.3 & 0.1 & 0.5 & 1.3 \\
\hline Surplus Electricity Credit ( $\phi / G G E)$ & 7 & 2 & 21 & -3 \\
\hline \multicolumn{5}{|l|}{ Fuel Blendstock Production Efficiencies } \\
\hline Biomass Feedstock (\%, LHV basis) & 47 & 43.6 & 43.6 & 48 \\
\hline Biomass + Natural Gas ${ }^{d}(\%$, LHV basis) & 47 & 43.6 & 43.4 & 47.4 \\
\hline $\begin{array}{l}\text { Biomass + Natural Gas }{ }^{d}+\text { Electricity }(\% \text {, LHV basis, } \\
\text { all electrical energy converted to heat) }\end{array}$ & 49 & 44.1 & 48.1 & 47.2 \\
\hline
\end{tabular}

${ }^{a}$ Updated from https://www.nrel.gov/docs/fy19osti/71954.pdf. ${ }^{\mathrm{b}}$ Fast pyrolysis intermediate yields maintained from 2015 design report (https://www.nrel.gov/docs/fy15osti/62455.pdf). ${ }^{c}$ Coke value is rounded up, and model assumption is higher than experimental result. ${ }^{d}$ Small amount of NG. ${ }^{e}$ Model separation \& distribution may differ from experiments because of differences in configurations and other assumptions. Parameters with greatest economic impacts are matched closely. ${ }^{f}$ Additional $>10 \%$ carbon in condensables not shown. ${ }^{g}$ Co-hydroprocessing. Abbreviations: $\mathrm{NG}=$ natural gas; GGE = gallon gasoline equivalent; LHV = lower heating value. 
Table ES-2. Economic Summary (Modeled) for the Updated 2018 SOT

Process Engineering Analysis for Hydrocarbon Fuel Production via Ex Situ Upgrading of Fast Pyrolysis Vapors

Potential Research-Driven Pathway for Cost-Competitiveness by 2022 2,000 Dry Metric Tonnes Biomass per Day

All Values in $2016 \$$

Minimum Fuel Selling Price (MFSP)

Gasoline Blendstock Production

Diesel Blendstock Production Total Gasoline Equivalent Production

Delivered Feedstock Cost Internal Rate of Return (After-Tax) Equity Percent of Total Investment On-Stream Factor

Capital Costs

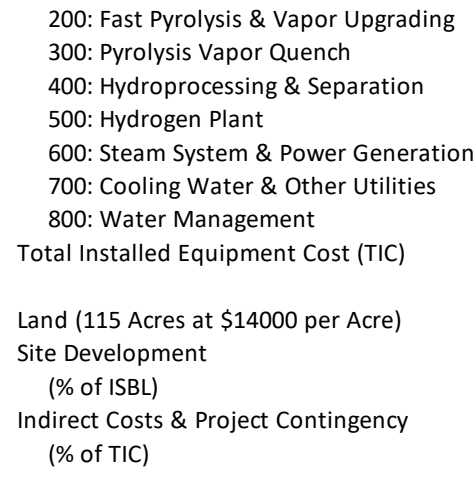

\$3.64 /Gallon Gasoline Blendstock

$\$ 4.14$ /Gallon Diesel Blendstock \$3.80 /Gallon Gasoline Equivalent (GGE)

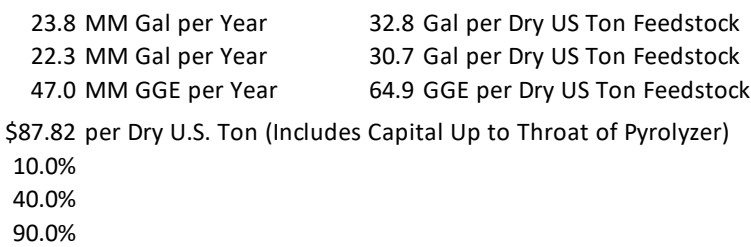

\begin{tabular}{lr}
\multicolumn{2}{c}{ Operating Costs (c / GGE Product) } \\
\hline Feedstock & 134.3 \\
Natural Gas & 0.4 \\
Catalysts & 16.8 \\
Sand & 0.6 \\
Other Raw Materials & 1.4 \\
Waste Disposal & 2.2 \\
Purchased Electricity & - \\
Fixed Costs & 60.0 \\
Electricity Coproduct Credit & $(7.5)$ \\
Capital Depreciation & 62.3 \\
Average Income Tax & 13.2 \\
Average Return on Investment & 95.8
\end{tabular}

\begin{tabular}{|c|c|c|}
\hline \multicolumn{3}{|c|}{ Operating Costs (\$ / Year) } \\
\hline \multicolumn{2}{|l|}{ Feedstock } & $\$ 63,100,000$ \\
\hline \multicolumn{2}{|l|}{ Natural Gas } & $\$ 200,000$ \\
\hline \multicolumn{2}{|l|}{ Catalysts } & $\$ 7,900,000$ \\
\hline \multicolumn{2}{|l|}{ Sand } & $\$ 300,000$ \\
\hline \multicolumn{2}{|l|}{ Other Raw Materials } & $\$ 680,000$ \\
\hline \multicolumn{2}{|l|}{ Waste Disposal } & $\$ 1,040,000$ \\
\hline \multicolumn{2}{|l|}{ Purchased Electricity } & $\$ 0$ \\
\hline \multicolumn{2}{|l|}{ Fixed Costs } & $\$ 28,200,000$ \\
\hline \multicolumn{2}{|c|}{ Electricity Coproduct Credit } & $-\$ 3,500,000$ \\
\hline \multicolumn{2}{|l|}{ Capital Depreciation } & $\$ 29,290,000$ \\
\hline \multicolumn{2}{|l|}{ Average Income Tax } & $\$ 6,210,000$ \\
\hline \multicolumn{2}{|c|}{ Average Return on Investment } & $\$ 45,010,000$ \\
\hline \multicolumn{2}{|c|}{ Total Plant Electricity Usage (kW) } & 44,446 \\
\hline \multicolumn{2}{|c|}{ Electricity Produced on Site (kW) } & 52,038 \\
\hline \multicolumn{2}{|c|}{ Electricity Purchased from Grid (kW) } & 0 \\
\hline \multicolumn{2}{|c|}{ Electricity Sold to Grid (kW) } & 7,593 \\
\hline Plant Electricity Use & (kWh /GGE) & 7.46 \\
\hline \multicolumn{3}{|c|}{ Specific Operating Conditions } \\
\hline \multirow[t]{2}{*}{ Feed Rate } & Dry Tonnes / Day & 2,000 \\
\hline & Dry Tons / Day & 2,205 \\
\hline \multirow[t]{2}{*}{ Feedstock Cost } & \$/Dry Ton & $\$ 87.82$ \\
\hline & $\$ /$ Moisture+Ash Free Ton & $\$ 88.64$ \\
\hline
\end{tabular}




\section{Table ES-3. Economic Summary (Modeled) for the 2019 SOT}

\section{Process Engineering Analysis for Hydrocarbon Fuel Production}

via Ex Situ Upgrading of Fast Pyrolysis Vapors

Potential Research-Driven Pathway for Cost-Competitiveness by 2022 2,000 Dry Metric Tonnes Biomass per Day

All Values in $2016 \$$

Minimum Fuel Selling Price (MFSP)

Gasoline Blendstock Production Diesel Blendstock Production Total Gasoline Equivalent Production Light Oxygenated Coproducts

Delivered Feedstock Cost Internal Rate of Return (After-Tax) On-Stream Factor

\begin{tabular}{|c|c|}
\hline 100: Feedstock (Additional Dryer \& Blower Only) & $\$ 520,000$ \\
\hline 200: Fast Pyrolysis \& Vapor Upgrading & $\$ 112,690,000$ \\
\hline 300: Vapor Quench \& CFP Co-Products & $\$ 30,430,000$ \\
\hline 400: Hydroprocessing \& Separation & $\$ 29,250,000$ \\
\hline 500: Hydrogen Plant & $\$ 69,300,000$ \\
\hline 600: Steam System \& Power Generation & $\$ 47,280,000$ \\
\hline 700: Cooling Water \& Other Utilities & $\$ 8,770,000$ \\
\hline 800: Water Management & $\$ 24,250,000$ \\
\hline Total Installed Equipment Cost (TIC) & $\$ 322,470,000$ \\
\hline Land (115 Acres at $\$ 14000$ per Acre) & $\$ 1,600,000$ \\
\hline Site Development & $\$ 18,120,000$ \\
\hline (\% of ISBL) & $10.0 \%$ \\
\hline Indirect Costs \& Project Contingency & $\$ 218,570,000$ \\
\hline (\% of TIC) & $66.1 \%$ \\
\hline Fixed Capital Investment (FCI) & $\$ 582,860,000$ \\
\hline Working Capital & $\$ 29,140,000$ \\
\hline Total Capital Investment (TCI) & $\$ 612,000,000$ \\
\hline Loan Interest Rate & $8.0 \%$ \\
\hline Loan Term (Years) & 10 \\
\hline Total Installed Equipment Cost per Annual GGE & $\$ 7.48$ \\
\hline Fixed Capital Investment per Annual GGE & $\$ 13.53$ \\
\hline Plant Operating Hours per Year & 7884 \\
\hline On-Stream Percentage & $90.0 \%$ \\
\hline \multicolumn{2}{|l|}{ Maximum Yield Based on HHV of Feedstock + Natural Gas } \\
\hline Theoretical GGE Production (MM GGE / Year) & 103.3 \\
\hline Theoretical Yield (GGE / Dry Ton) & 142.6 \\
\hline Current Yield (Actual / Theoretical) & $41.7 \%$ \\
\hline Overall Plant Efficiency - HHV \% & $43.3 \%$ \\
\hline Overall Plant Efficiency - LHV \% & $43.6 \%$ \\
\hline
\end{tabular}

\$3.21 /Gallon Gasoline Blendstock \$3.63 /Gallon Diesel Blendstock \$3.33 /Gallon Gasoline Equivalent (GGE)

23.1 MM Gal per Year 19.1 MM Gal per Year 43.1 MM GGE per Year 49.1 MM lb per year $2.7 \%$ Acetone/Dry Biomass 31.9 Gal per Dry US Ton Feedstock 26.4 Gal per Dry US Ton Feedstock 59.5 GGE per Dry US Ton Feedstock $3.4 \% \mathrm{w} / \mathrm{w}$ of Dry Biomass $0.6 \%$ MEK/Dry Biomass $\$ 70.15$ per Dry U.S. Ton (Includes Capital Up to Throat of Pyrolyzer) $10.0 \%$ Equity, \% of Total Investment $40.0 \%$ $90.0 \%$

\begin{tabular}{lr}
\multicolumn{2}{c}{ Operating Costs (c/ GGE Product) } \\
\hline Feedstock & 117.0 \\
Natural Gas & 0.1 \\
Catalysts & 14.2 \\
Sand & 0.6 \\
Other Raw Materials & 1.6 \\
Waste Disposal & 3.3 \\
Purchased Electricity & - \\
Fixed Costs & 65.2 \\
Chemical Coproduct Credit & $(51.7)$ \\
Electricity Coproduct Credit & $(2.1)$ \\
Capital Depreciation & 67.6 \\
Average Income Tax & 14.2 \\
Average Return on Investment & 102.9
\end{tabular}

\begin{tabular}{|c|c|c|}
\hline \multicolumn{3}{|c|}{ Operating Costs (\$ / Year) } \\
\hline Feedstock & & $\$ 50,410,000$ \\
\hline Natural Gas & & $\$ 0$ \\
\hline Catalysts & & $\$ 6,130,000$ \\
\hline Sand & & $\$ 300,000$ \\
\hline Other Raw Mat & & $\$ 700,000$ \\
\hline Waste Disposal & & $\$ 1,420,000$ \\
\hline Purchased Elect & & $\$ 0$ \\
\hline Fixed Costs & & $\$ 28,090,000$ \\
\hline Chemical Copro & Credit & $-\$ 22,290,000$ \\
\hline Electricity Coprc & Credit & $-\$ 920,000$ \\
\hline Capital Depreci & & $\$ 29,140,000$ \\
\hline Average Income & & $\$ 6,110,000$ \\
\hline Average Return & vestment & $\$ 44,320,000$ \\
\hline Total Plant Elec & Usage $(\mathrm{kW})$ & 40,542 \\
\hline Electricity $\operatorname{Pr}$ & $\mathrm{d}$ on Site (kW) & 42,540 \\
\hline Electricity $\mathrm{Pu}$ & ed from Grid (kW) & 0 \\
\hline Electricity So & Grid $(k W)$ & 1,997 \\
\hline Plant Electricity & (kWh /GGE) & 7.42 \\
\hline \multicolumn{3}{|c|}{ Specific Operating Conditions } \\
\hline \multirow[t]{2}{*}{ Feed Rate } & Dry Tonnes / Day & 2,000 \\
\hline & Dry Tons / Day & 2,205 \\
\hline \multirow[t]{2}{*}{ Feedstock Cost } & $\$ /$ Dry Ton & $\$ 70.15$ \\
\hline & $\$ /$ Moisture+Ash Free Ton & $\$ 71.39$ \\
\hline
\end{tabular}




\section{Table ES-4. Economic Summary (Modeled) for the 2020 Projection with Co-Hydroprocessing Option}

\section{Process Engineering Analysis for Hydrocarbon Fuel Production via Ex Situ Upgrading of Fast Pyrolysis Vapors}

Potential Research-Driven Pathway for Cost-Competitiveness by 2022 2,000 Dry Metric Tonnes Biomass per Day All Values in $2016 \$$

Minimum Fuel Selling Price (MFSP)

Gasoline Blendstock Production Diesel Blendstock Production Total Gasoline Equivalent Production Catalytic Fast Pyrolysis Oil Light Oxygenated Coproducts

Delivered Feedstock Cost Internal Rate of Return (After-Tax) On-Stream Factor

200: Fast Pyrolysis \& Vapor Upgrading 300: Vapor Quench \& CFP Co-Products 400: Hydroprocessing \& Separation 500: Hydrogen Plant

600: Steam System \& Power Generation 700: Cooling Water \& Other Utilities 800: Water Management

Total Installed Equipment Cost (TIC)

Land (115 Acres at \$14000 per Acre)

Site Development

(\% of ISBL)

Indirect Costs \& Project Contingency (\% of TIC)

Fixed Capital Investment (FCl) Working Capital

Total Capital Investment (TCI)

$\$ 26,540,000$

$\$ 557,280,000$

Loan Interest Rate

Loan Term (Years)

$8.0 \%$

10

Total Installed Equipment Cost per Annual GGE

Fixed Capital Investment per Annual GGE

$\$ 6.65$

$\$ 12.32$

Plant Operating Hours per Year

On-Stream Percentage

7884

$90.0 \%$

Maximum Yield Based on HHV of Feedstock + Natural Gas Theoretical GGE Production (MM GGE / Year)

Theoretical Yield (GGE / Dry Ton)

Current Yield (Actual / Theoretical)

103.8

143.3

$41.5 \%$

Overall Plant Efficiency - HHV \%

Overall Plant Efficiency - LHV \%
$43.1 \%$

$43.4 \%$
Capital Costs

\section{\$2.98 /Gallon Gasoline Blendstock \\ \$3.37 /Gallon Diesel Blendstock \\ \$3.09 /Gallon Gasoline Equivalent (GGE)}

23.1 MM Gal per Year

19.1 MM Gal per Year

31.9 Gal per Dry US Ton Feedstock

43.1 MM GGE per Year

26.4 Gal per Dry US Ton Feedstock

$0.95 \mathrm{MM}$ bbl per Year

59.5 GGE per Dry US Ton Feedstock

$49.1 \mathrm{MM}$ Ib per year

$1.3 \mathrm{bbl}$ per Dry US Ton Feedstock

$\$ 70.15$ per Dry U.S. Ton (Includes Capital Up to Throat of Pyrolyzer)

$10.0 \%$ Equity, \% of Total Investment $40.0 \%$

90.0\% CFP Oil (\$/bbl GE Product) $\$ 120.78$

\begin{tabular}{lr}
\multicolumn{2}{c}{ Operating Costs (c/ GGE Product) } \\
\hline Feedstock & 117.0 \\
Natural Gas & 0.8 \\
Catalysts & 8.2 \\
Sand & 0.6 \\
Other Raw Materials & 1.5 \\
Waste Disposal & 3.1 \\
Purchased Electricity & - \\
Fixed Costs & 60.7 \\
Refinery Coprocessing Cost & 21.3 \\
Chemical Coproduct Credit & $(51.7)$ \\
Electricity Coproduct Credit & $(21.4)$ \\
Capital Depreciation & 61.6 \\
Average Income Tax & 13.1 \\
Average Return on Investment & 94.0
\end{tabular}

\begin{tabular}{lr}
\multicolumn{2}{c}{ Operating Costs (\$ / Year) } \\
\hline Feedstock & $\$ 50,410,000$ \\
Natural Gas & $\$ 400,000$ \\
Catalysts & $\$ 3,550,000$ \\
Sand & $\$ 300,000$ \\
Other Raw Materials & $\$ 660,000$ \\
Waste Disposal & $\$ 1,350,000$ \\
Purchased Electricity & $\$ 0$ \\
Fixed Costs & $\$ 26,160,000$ \\
Refinery Coprocessing Cost & $\$ 9,160,000$ \\
Chemical Coproduct Credit & $-\$ 22,290,000$ \\
Electricity Coproduct Credit & $-\$ 9,240,000$ \\
Capital Depreciation & $\$ 26,540,000$ \\
Average Income Tax & $\$ 5,650,000$ \\
Average Return on Investment & $\$ 40,490,000$ \\
& \\
Total Plant Electricity Usage (kW) & 38,981 \\
$\quad$ Electricity Produced on Site (kW) & 59,012 \\
$\quad$ Electricity Purchased from Grid (kW) & 0 \\
$\quad$ Electricity Sold to Grid (kW) & 20,031
\end{tabular}

Plant Electricity Use (kWh /GGE)

7.13

Version:

PyVPU-v218h ES FixedBed-v49-r046-AP10-FY20Target-V18-Acetone-MEK-HTcoproc-03

\begin{tabular}{llr}
\multicolumn{2}{c}{ Specific Operating Conditions } \\
\hline Feed Rate & Dry Tonnes / Day & 2,000 \\
& Dry Tons / Day & 2,205 \\
Feedstock Cost & \$/Dry Ton & $\$ 70.15$ \\
& \$/Moisture+Ash Free Ton & $\$ 71.39$
\end{tabular}




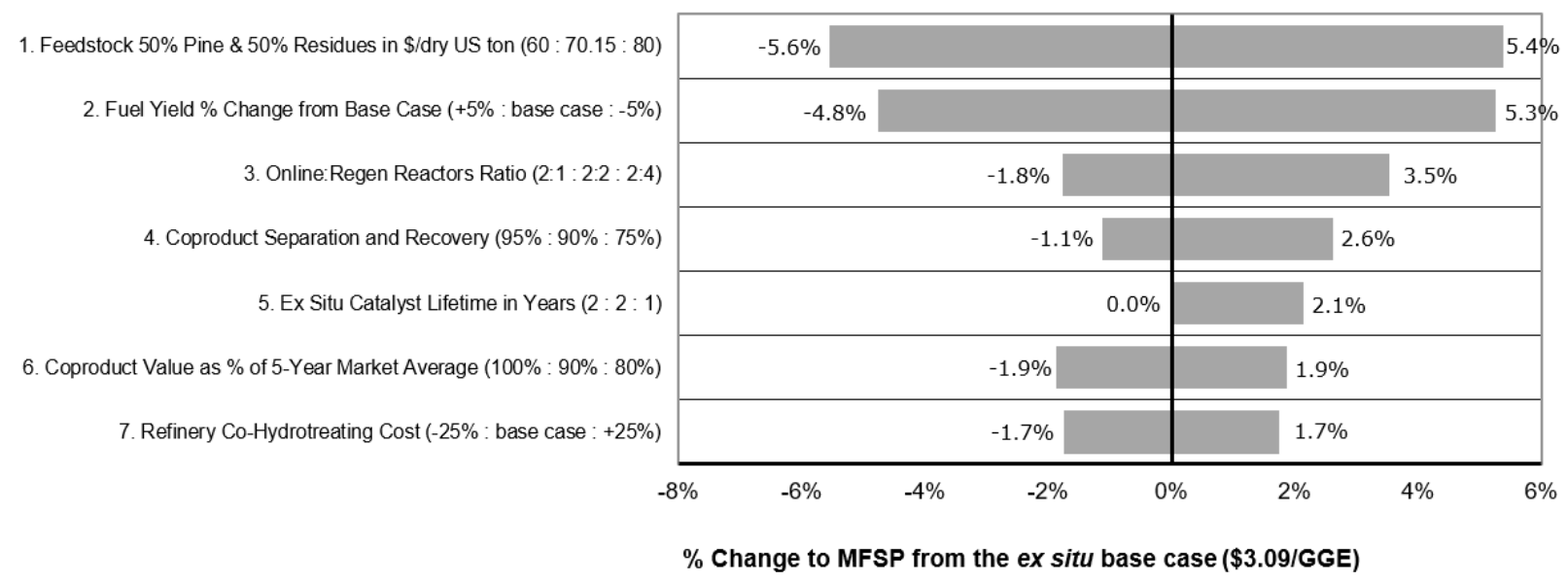

Figure ES-2. Sensitivity analysis for the $\mathbf{2 0 2 0}$ model with coproducts and co-hydroprocessing 


\section{Table ES-5. Economic Summary (Modeled) for the 2020 Projection with Yield Increase Option}

\section{Process Engineering Analysis for Hydrocarbon Fuel Production}

via Ex Situ Upgrading of Fast Pyrolysis Vapors

Potential Research-Driven Pathway for Cost-Competitiveness by 2022

2,000 Dry Metric Tonnes Biomass per Day

All Values in $2016 \$$

Minimum Fuel Selling Price (MFSP)

Gasoline Blendstock Production Diesel Blendstock Production Total Gasoline Equivalent Production Light Oxygenated Coproducts

Delivered Feedstock Cost Internal Rate of Return (After-Tax) On-Stream Factor

100: Feedstock (Additional Dryer \&
200: Fast Pyrolysis \& Vapor Upgradi
300: Vapor Quench \& CFP Co-Products
400: Hydroprocessing \& Separation
500: Hydrogen Plant
600: Steam System \& Power Genera
700: Cooling Water \& Other Utilitie
800: Water Management
Total Installed Equipment Cost (TIC)
Land (115 Acres at \$14000 per Acre)
Site Development
(\% of ISBL)
Indirect Costs \& Project Contingency
(\% of TIC)
Fixed Capital Investment (FCI)
Working Capital
Total Capital Investment (TCI)
Loan Interest Rate
Loan Term (Years)

Total Installed Equipment Cost per Annual GGE

Fixed Capital Investment per Annual GGE

Plant Operating Hours per Year

On-Stream Percentage

Maximum Yield Based on HHV of Feedstock + Natural Gas

Theoretical GGE Production (MM GGE / Year)

Theoretical Yield (GGE / Dry Ton)

Current Yield (Actual / Theoretical)

Overall Plant Efficiency - HHV \%

Overall Plant Efficiency - LHV \%

Version:

PyVPU-v218h ES FixedBed-v49-r046-AP10-FY20Target-V18-Acetone-MEK-3pct-higher-C-eff-03-\$3.09

25.6 MM Gal per Year

20.8 MM Gal per Year

47.4 MM GGE per Year

48.7 MM lb per year $90.0 \%$

\$2.98 /Gallon Gasoline Blendstock

\$3.38 /Gallon Diesel Blendstock \$3.09 /Gallon Gasoline Equivalent (GGE)

$2.7 \%$ Acetone/Dry Biomass

35.4 Gal per Dry US Ton Feedstock $28.8 \mathrm{Gal}$ per Dry US Ton Feedstock 65.5 GGE per Dry US Ton Feedstock $3.4 \% \mathrm{w} / \mathrm{w}$ of Dry Biomass $0.6 \%$ MEK/Dry Biomass $\$ 70.15$ per Dry U.S. Ton (Includes Capital Up to Throat of Pyrolyzer) $10.0 \%$ Equity, \% of Total Investment $40.0 \%$

\begin{tabular}{lr}
\multicolumn{2}{c}{ Operating Costs (c / GGE Product) } \\
\hline Feedstock & 106.3 \\
Natural Gas & 1.9 \\
Catalysts & 13.5 \\
Sand & 0.6 \\
Other Raw Materials & 1.5 \\
Waste Disposal & 3.0 \\
Purchased Electricity & - \\
Fixed Costs & 59.2 \\
Chemical Coproduct Credit & $(46.6)$ \\
Electricity Coproduct Credit & 2.5 \\
Capital Depreciation & 61.4 \\
Average Income Tax & 12.8 \\
Average Return on Investment & 93.4
\end{tabular}

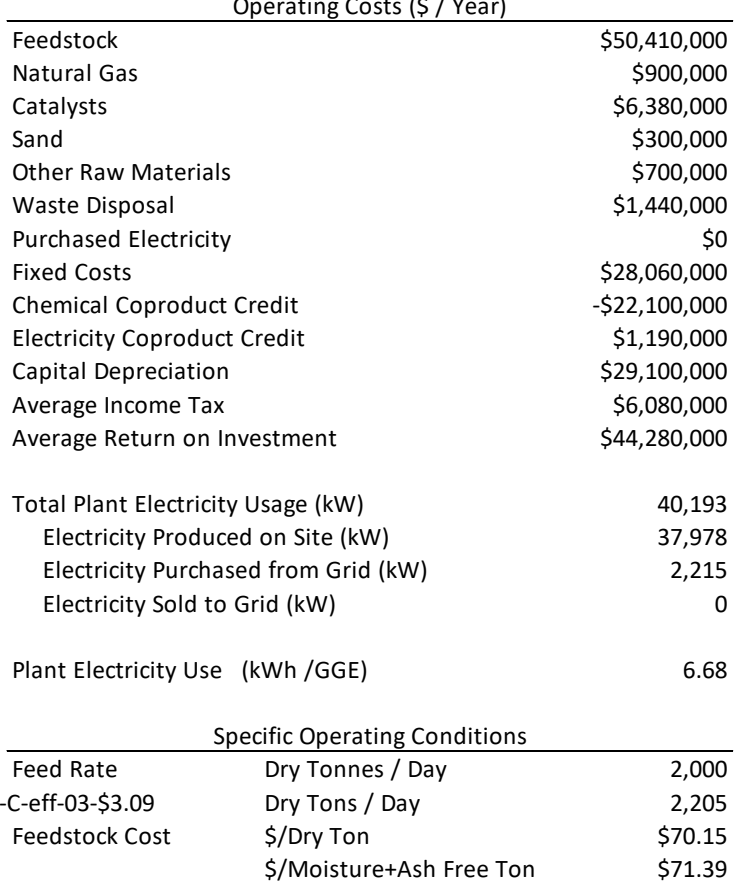

xvii 


\section{Table of Contents}

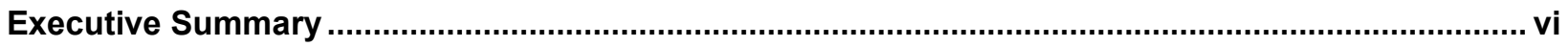

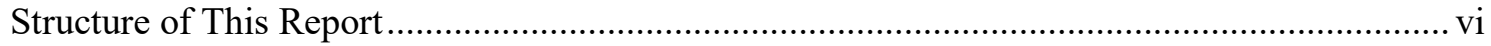

Background Information ………………………………………………………………... vi

Research and Development Since the 2018 SOT Report......................................................... vii

Research Plans................................................................................................................... ix

Summary of Key Results.......................................................................................................

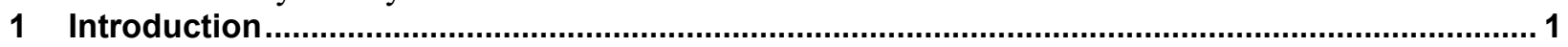

1.1 Techno-Economic Analysis Approach..................................................................................... 1

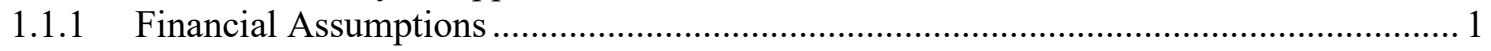

1.1.2 Estimation of Capital and Operating Costs …………………………………………2

1.1.3 Minimum Fuel Selling Price ...................................................................................

1.1.4 The Process Model .............................................................................................

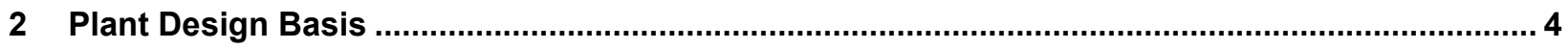

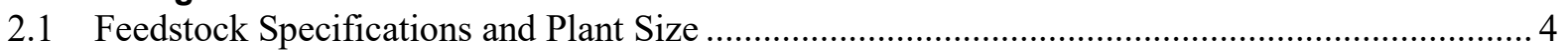

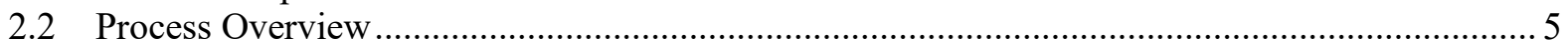

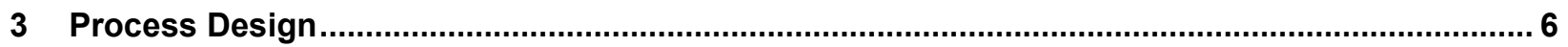

3.1 Area 100: Feed Handling …………………………...................................................

3.2 Area 200: Fast Pyrolysis and Catalytic Vapor Upgrading ......................................................... 7

3.3 Area 300: CFP Product Condensation and Coproducts ............................................................ 8

3.4 Area 400: CFP Oil Hydroprocessing ............................................................................... 9

3.5 Area 500: Hydrogen Production........................................................................................ 9

3.6 Area 600: Steam System and Electricity Generation ................................................................. 9

3.7 Area 700: Cooling Water and Other Utilities........................................................................ 9

3.8 Area 800: Wastewater Utilization and Treatment ................................................................... 9

$3.9 \quad$ Process Heat Exchange Cost ………………………........................................................

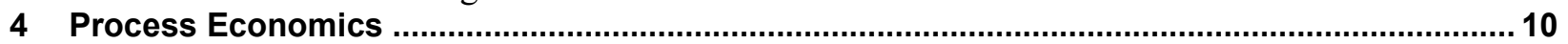

$4.1 \quad$ Total Capital Investment ............................................................................................. 10

4.2 Operating Costs .............................................................................................................. 12

4.3 Discounted Cash Flow Analysis and the Minimum Fuel Selling Price ..................................... 13

$4.4 \quad$ Value of Hydrocarbon Fuel Products ........................................................................................ 13

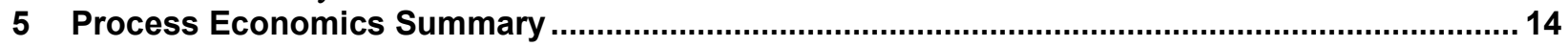

6 Sustainability and Life Cycle Analysis …………….............................................................. 16

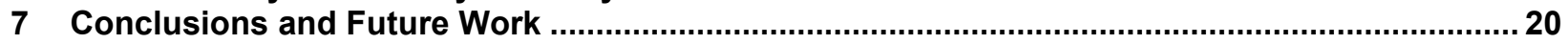

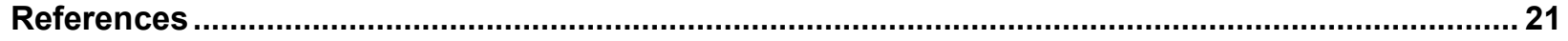

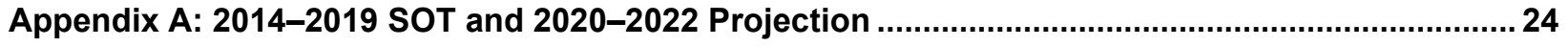




\section{List of Figures}

Figure ES-1. Modeled MFSP for 2014-2019 SOTs and 2020-2022 projections....................................... Figure ES-2. Sensitivity analysis for the 2020 model with coproducts and co-hydroprocessing .............. xvi Figure 1. Simplified process flow diagram for fixed bed ex situ catalytic fast pyrolysis, coproduct

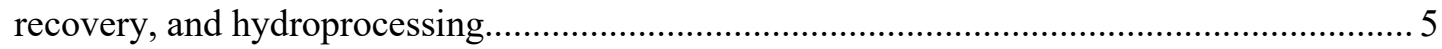

Figure 2. Cost contribution details from each process area for the 2019 SOT ...................................... 14

Figure 3. Cost contribution details from each process area for the 2020 co-HP projection ...................... 14

\section{List of Tables}

Table ES-1. Key Metrics for Updated Fiscal Year (FY) 2018 SOT, 2019 SOT, and 2020 Projections.....xii

Table ES-2. Economic Summary (Modeled) for the Updated 2018 SOT ...............................................iii

Table ES-3. Economic Summary (Modeled) for the 2019 SOT ................................................................

Table ES-4. Economic Summary (Modeled) for the 2020 Projection with Co-Hydroprocessing Option.. XV

Table ES-5. Economic Summary (Modeled) for the 2020 Projection with Yield Increase Option...........xvii

Table 1. Summary of Financial Assumptions for Techno-Economic Analysis ......................................... 2

Table 2. INL Modeled Feedstock Cost for 50\% Forest Residues Plus 50\% Clean Pine in 2016\$ (used for 2019-2020 models)

Table 3. INL Modeled Feedstock Cost for 75\% Forest Residues Plus 25\% Clean Pine in 2016\$ (alternate mix used for the 2021-2022 projection models)

Table 4. Total Installed Equipment Costs for the 2019 SOT and 2020 Projection with Co-HP................ 10

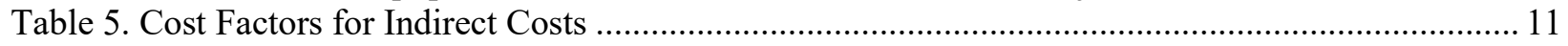

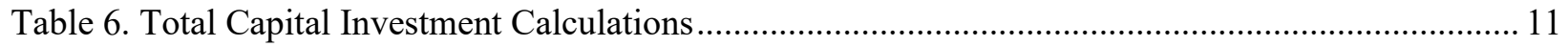

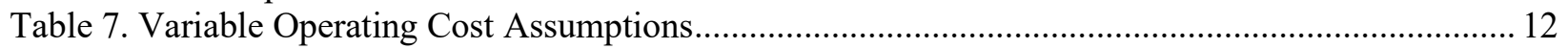

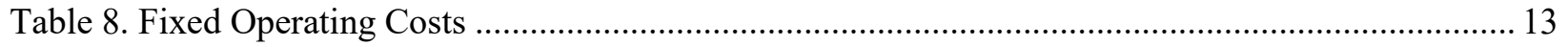

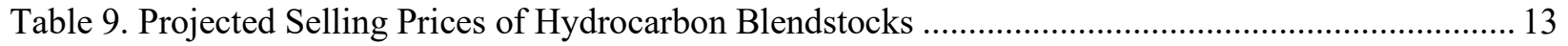

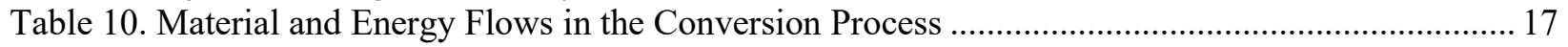

Table 11. Sustainability and Process Efficiency Metrics for the Conversion Process............................... 18

Table A-1. Processing Area Cost Contributions and Key Technical Parameters ..................................... 24 


\section{Introduction}

The 2015 catalytic fast pyrolysis (CFP) design report [1] detailed in situ and ex situ CFP as two potential research options for the conversion of biomass to liquid transportation fuels. Catalysts are included within the fast pyrolysis reactor in an in situ process. On the other hand, biomassderived solid material (char and inorganic matter) are separated after fast pyrolysis in an ex situ process; removal of all solids from pyrolysis vapors is desirable before catalytic upgrading in an ex situ reactor. The 2015 design report helped outline the basis for technical improvements necessary for future economic viability; associated modeled costs were presented to help understand the potential value of the research improvements [2]. Since 2015, the focus of the research has been on ex situ systems because it affords more opportunity to understand and affect the chemistry through catalyst development in the absence of biomass-derived solid material present in an in situ system. Bench-scale experimental results have been used to update the state of technology (SOT) for the ex situ pathway since 2014; significant yield improvements have been achieved via catalyst development.

Ex situ research under this project was initiated on fluidized systems with zeolite-based (primarily ZSM-5 and metal impregnated ZSM-5) catalysts. This was based on historic precedence of experimental work documenting some of the best yields using ZSM-5 catalysts for catalytic fast pyrolysis [3]. Circulating fluidized bed systems with a combustor for coke burnoff are ideal for ZSM-5 catalysts. To broaden the research and explore other bifunctional catalyst options [4,5], such as those with noble metals in their formulations as one such option, an analogous fixed bed approach was proposed, and its feasibility was analyzed by Dutta et al. [6]. Consequent catalyst research and associated experimental performance showed significant yield improvements using a $\mathrm{Pt} / \mathrm{TiO}_{2}$ catalyst [7]. Hence, the current process configuration for the SOT experiments since 2017 and future projections were based on the $\mathrm{Pt} / \mathrm{TiO}_{2}$ catalyst in a fixed bed as the base configuration.

The Executive Summary provides information about our research findings since the 2018 SOT update [8]; those details are not repeated here.

\subsection{Techno-Economic Analysis Approach}

The techno-economic analysis (TEA) approach for this work is similar to those detailed previously $[1,6]$. Overviews of process and economic assumptions and methods are provided below. Further details are available in these previous process design reports $[1,6]$.

\subsubsection{Financial Assumptions}

The modeled projections in this report are based on the technology being implemented in a mature or $\mathrm{n}^{\text {th }}$ plant; additional costs associated with pioneer plants are thus not included because the purpose of this TEA is to understand the potential impact and relevance of the research in the context of future industrial implementation. A consistent set of assumptions is used for all SOT and projections. Key assumptions are listed in Table 1. 
Table 1. Summary of Financial Assumptions for Techno-Economic Analysis

\begin{tabular}{lr}
\hline Description of Assumption & Assumed Value \\
\hline Cost year & 2016 \\
Internal rate of return on equity & $10 \%$ \\
Plant financing by equity/debt & $40 \% / 60 \%$ of total capital investment \\
Plant life & 30 years \\
Income tax rate & $21 \%$ \\
Interest rate for debt financing & \\
Term for debt financing & $8.0 \%$ annually \\
Working capital cost & 10 years \\
Depreciation schedule & (excluding land purchase cost) \\
Steam plant depreciation & 7 -year MACRS schedule [9] \\
Construction period (spending schedule) & 20 -year MACRS schedule [9] \\
Plant salvage value & 3 years (8\% Y1, $60 \%$ Y $2,32 \%$ Y 3$)$ \\
Startup time & No value \\
Revenue and costs during startup & 6 months \\
& \\
Onstream percentage after startup & Revenue $=50 \%$ of normal \\
\hline MACRS = modified accelerated cost recovery system & $90 \%$ (7,884 operating hours per year) \\
\hline
\end{tabular}

There were two significant changes to the financial assumptions compared to the previous publications [1,6]: (1) $21 \%$ tax rate (versus a previous 35\% tax rate); and (2) 2016-dollars cost basis was used. These assumptions in Table 1 are consistent with the 2018 SOT report [8].

\subsubsection{Estimation of Capital and Operating Costs}

Detailed capital costs of individual equipment and their sources were listed in the 2015 design report [1], and additional fixed bed equipment costs were presented in the subsequent analysis for fixed bed systems $[6,8]$. Note that the fixed bed system cost for this analysis was based on the \#1 upstream reactor in Dutta et al. [6], at approximately $\$ 2.5$ million base cost per $50 \%$ capacity reactor in 2013 dollars, a scaling exponent of 0.7, and an installation factor of 1.62.

Equipment costs were scaled based on process flows in the Aspen Plus process model using a scaling exponent:

$$
\text { Scaled Equipment Cost }=\text { Base Equipment Cost }\left(\frac{\text { Scaled Capacity }}{\text { Base Capacity }}\right)^{n}
$$

The scaling exponent, $\mathrm{n}$, is typically in the range of 0.6 to 0.7 for process equipment; however, it varies with equipment type, base size, and with other factors that affect scalability. Scaling factors are documented in Appendix B of the 2015 design report [1]. 
Total installed cost (TIC) of the equipment, which includes associated piping, instrumentation and controls, electrical systems, buildings, yard improvements, and direct labor, were derived from the equipment cost by applying an installation factor $\left(f_{\text {installation }}\right)$.

Total Installed Cost $($ TIC $)=f_{\text {installation }} *$ Total Purchased Equipment Cost (TPEC)

Installation factors are also documented in Appendix B of the 2015 design report.

Costs were converted to 2016 dollars using:

$$
\text { Cost in } 2016 \$=\text { Base Cost }\left(\frac{2016 \text { Cost Index Value }}{\text { Base Year Cost Index Value }}\right)
$$

Operating costs were adjusted using the Producer Price Index for Chemical Manufacturing [10] and capital costs were adjusted using the Chemical Engineering's Plant Cost Index [11].

The total capital investment (TCI) was derived from the TIC in 2016 dollars after applying additional factors for overhead and contingency.

\subsubsection{Minimum Fuel Selling Price}

The TCI, along with plant operating costs, was used for a discounted cash flow analysis. Those costs along with the gallons gasoline equivalent (GGE) of the total fuel blendstock product were used to derive the minimum fuel selling price (MFSP) in \$/GGE.

\subsubsection{The Process Model}

The process was modeled in Aspen Plus with a detailed accounting of all mass and energy flows. Details about the Aspen Plus [12] process model for ex situ CFP were documented in the 2015 design report [1] and the subsequent fixed bed publication [6]. The base models from the previous work were maintained for this analysis. Process assumption updates and other key aspects are described in the following sections. 


\section{Plant Design Basis}

\subsection{Feedstock Specifications and Plant Size}

Feedstock information for this process was provided by Idaho National Laboratory (INL). Feedstock blends costs used for the 2019-2020 cases and proposed for the 2021-2022 projections (included in the Appendix) were both modeled at approximately \$70/dry US ton. The plant size was maintained at 2,000 dry metric tons per day.

The 2019 SOT feedstock was based on a 50/50 blend of forest residues and clean pine at $\$ 70.15 /$ dry U.S. ton in 2016 dollars with a relatively high $1.75 \%$ modeled ash content (also reflected in the conversion process model by a prorated reduction of the other elements in the elemental analysis). Another feedstock blend option is being considered by INL and the 20212022 feedstock cost projection is based on a blend of $75 \%$ air-classified forest residues and $25 \%$ clean pine, with additional cleanup steps for ash reduction to below $1 \%$; the modeled cost for this feedstock is $\$ 70.31$ dry U.S. ton in 2016 dollars. Given the low ash in this 2021-2022 feedstock option, the specification assumption in the process model was unaltered from the 2015 design report [1], with an elemental analysis of C:50.94\%, H:6.04\%, N:0.17\%, S:0.03\%, O:41.90\%, Ash: $0.92 \%$ on a dry basis, and $10 \%$ moisture at the plant gate.

Although the 2021-2022 models include a different blend in the current projections, the final determination of the feedstock to be used in 2021-2022 will be made based on the performance of the current 50/50 blend feedstock during the 500-hour run, as discussed in the Executive Summary. 


\subsection{Process Overview}

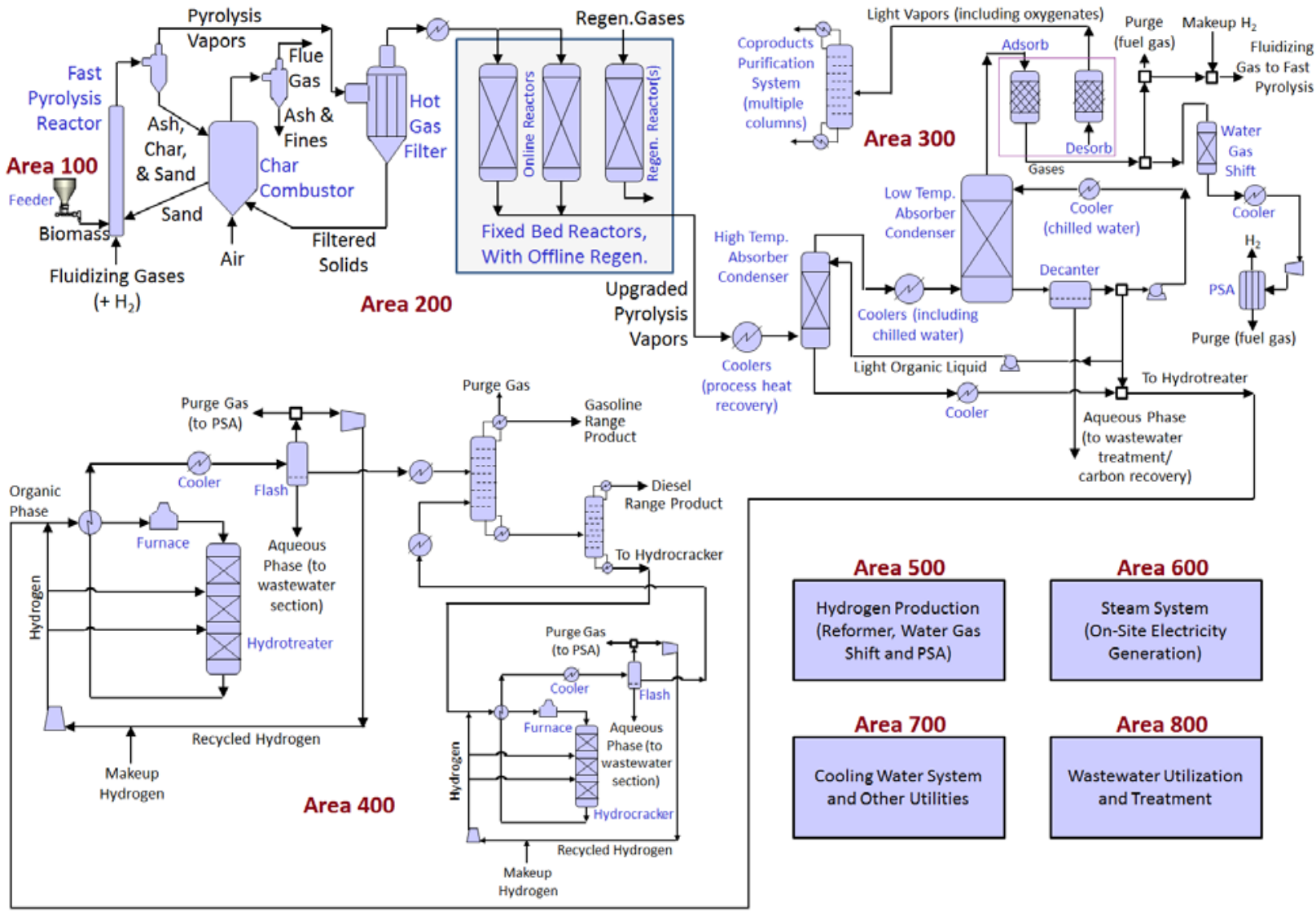

Figure 1. Simplified process flow diagram for fixed bed ex situ catalytic fast pyrolysis, coproduct recovery, and hydroprocessing

A block flow diagram for the fixed bed ex situ catalytic fast pyrolysis process is shown in Figure 1. The design includes eight process areas, with four core operations:

- A100: Feedstock Handling (most of the processing occurs off-site and the TEA accounts for the feedstock delivered to the throat of the reactor through a cumulative cost)

- A200: Fast Pyrolysis, Hot Gas Filtration, and Ex Situ Catalytic Vapor Upgrading

- A300: CFP Product Condensation (with the separation of the organic liquid CFP oil from an aqueous wastewater stream and use of separated permanent gases in the process). Beginning 2019, oxygenated coproducts recovery and purification was added to this area

- A400: CFP Oil Hydrotreating, Hydrocracking, and Product Distillation.

Supporting operations include:

- A500: Hydrogen Production (from process off-gases)

- A600: Steam System and Electricity Generation (from available excess heat)

- A700: Cooling Water and Other Utilities

- A800: Wastewater Utilization and Treatment (regenerative thermal oxidizer used to combust the organic content in the wastewater).

Further descriptions are included in Section 3. 


\section{Process Design}

\subsection{Area 100: Feed Handling}

As mentioned in Section 2.1, two different blends of forest residues and clean pine were considered in the 2019-2020 and 2021-2022 TEA models; blending forest residues with clean pine enables lower costs. The moisture content was $10 \%$ in both cases, but with a higher modeled ash content of $1.75 \%$ in the $2019-2020$ cases. All feedstock growth, handling, and processing costs are included in the cumulative feedstock costs presented below. A nominal feedstock size of $2 \mathrm{~mm}$ is specified for this process and necessary grinding costs are included in INL's feedstock cost [13]. The only minor feedstock handling-related cost added to the plant equipment is a cross-flow dryer for warming the feedstock before feeding to the fast pyrolysis reactor.

Currently, it is estimated that there are 21.2 million dry U.S. tons of pine feedstocks available nationally; 11.8 million dry U.S. tons are planted pine and 9.4 million dry U.S. tons are pine forest residues. This amount of material can be aggregated to support approximately 25 biorefineries of 2,000 dry metric tons per day, given no competition for the resource [13]; however, not all material would be available within the required cost envelope to enable the current cost targets.

The modeled cost summary for a lower cost 2019-2020 feedstock with a mix of 50\% forest residues and 50\% clean pine with an aggregated ash content of $1.75 \%$ (modeled) is presented in Table 2 .

Table 2. INL Modeled Feedstock Cost for 50\% Forest Residues Plus 50\% Clean Pine in $2016 \$$ (used for 2019-2020 models)

\begin{tabular}{|l|l|}
\hline \multicolumn{2}{|l|}{ Cost Summary (\$/Dry U.S. Ton) } \\
\hline & 2016\$) \\
\hline Grower Payment & $\$ 9.74$ \\
\hline Harvest and Collection & $\$ 4.94$ \\
\hline Field-Side Preprocessing & $\$ 8.41$ \\
\hline Transportation & $\$ 12.22$ \\
\hline Preprocessing & $\$ 28.55$ \\
\hline Storage & $\$ 0.68$ \\
\hline Handling & $\$ 2.65$ \\
\hline Preprocessing Construction & $\$ 2.96$ \\
\hline Quality Dockage & $\$ 0.00$ \\
\hline Grand Total & $\$ 70.15$ \\
\hline
\end{tabular}

An alternate feedstock using a mix of clean pine and forest residues was modeled. This scenario estimates a total of 35.9 million U.S. tons available nationwide. If we consider only the volume that is aggregable within a 725,000 dry U.S. ton supply shed (necessary for a 2,000-dry-metric- 
tons-per-day plant operating at a $90 \%$ onstream factor) and ignore "stranded" resources, there is enough forest residue to supply 17 biorefineries at a size of 2,000 dry metric tons per day; however, the quality of higher blend levels of forest residues may be problematic for the conversion process because of a higher overall ash and alkali and alkaline earth metal (AAEM) concentrations compared to clean pine. If necessary, additional cleaning steps can help reduce this ash content. Cleaning steps are detailed in $\mathrm{Hu}$ et al. [14]. These steps can reduce the ash content below 1\%. Experimental and model results will be used to co-optimize feedstock cost and quality, performance during the conversion process, and the impact of feedstock preprocessing steps on the LCA. The modeled cost summary for a 2022 feedstock option with $75 \%$ air-classified forest residues and $25 \%$ clean pine is presented in Table 3.

Table 3. INL Modeled Feedstock Cost for $75 \%$ Forest Residues Plus $25 \%$ Clean Pine in $2016 \$$ (alternate mix used for the 2021-2022 projection models)

\begin{tabular}{|c|c|}
\hline \multicolumn{2}{|c|}{ Cost Summary (\$/Dry U.S. Ton) (2016\$) } \\
\hline & 2022 Projection \\
\hline Grower Payment & $\$ 6.84$ \\
\hline Harvest and Collection & $\$ 2.47$ \\
\hline Field-Side Preprocessing & $\$ 9.81$ \\
\hline Transportation & $\$ 13.88$ \\
\hline Preprocessing & $\$ 31.63$ \\
\hline Storage & $\$ 0.58$ \\
\hline Handling & $\$ 1.90$ \\
\hline Preprocessing Construction & $\$ 3.21$ \\
\hline Quality Dockage & $\$ 0.00$ \\
\hline Grand Total & $\$ 70.31$ \\
\hline
\end{tabular}

\subsection{Area 200: Fast Pyrolysis and Catalytic Vapor Upgrading}

The process model for Area 200 includes a circulating fluidized bed fast pyrolysis reactor. The dual-bed reactor system includes a riser reactor for fast pyrolysis of biomass at approximately $500^{\circ} \mathrm{C}\left(932^{\circ} \mathrm{F}\right)$, with short biomass residence times of approximately 2 seconds in the riser and a char combustor for providing heat to the endothermic fast pyrolysis reactions; circulating sand is heated in the char combustor and sent to the riser reactor where it heats the biomass to pyrolysis temperatures. The solids (char and mineral matter) from fast pyrolysis are removed from the hot vapors by cyclones. An additional hot gas filter (HGF) is also included to remove any residual solids. This HGF is necessary because of the downstream fixed bed ex situ catalytic vapor upgrading reactor that can easily plug from any residual solids. The catalytic fixed bed reactor system includes a $\mathrm{Pt} / \mathrm{TiO}_{2}$ catalyst with $0.5 \mathrm{wt} \% \mathrm{Pt}$ loading. A 2-year catalyst lifetime is assumed in the model, along with a $70 \%$ cost recovery at the end of 2 years. A catalyst cost model, called CatCost, [15] developed under the Chemical Catalysis for Bioenergy Consortium [16] was used to estimate the cost of the $\mathrm{Pt} / \mathrm{TiO}_{2}$ catalyst. Note that the 2015 design report [1] included a circulating fluidized bed ex situ reactor design with zeolite catalyst. 
The CFP bench-scale experimental setup and analytical methods used to generate experimental results for the 2018 SOT are described by Griffin et al. [7] and in the 2018 SOT report [8]. As a brief overview, a 2-inch fluidized pyrolyzer was followed by an $\mathrm{HGF}$ and a fixed-bed $\mathrm{Pt} / \mathrm{TiO}_{2}$ catalyst vapor upgrading reactor.

The $50 \%$ forest residues $/ 50 \%$ clean pine blend was converted at a fast pyrolysis temperature of $500^{\circ} \mathrm{C}$, the fixed bed ex situ reactor setpoint temperature was maintained at $400^{\circ} \mathrm{C}$, and the biomass:catalyst $(\mathrm{B}: \mathrm{C})$ ratio was 12 . The catalyst had $0.5 \mathrm{wt} \% \mathrm{Pt}$ on a $\mathrm{TiO}_{2}$ support. CFP oil with an oxygen content of $15 \mathrm{wt} \%$ on dry basis was produced with a carbon yield of $35 \%$. Compared to the FY18 SOT, the CFP oil had a lower oxygen content, as intended ( $15 \mathrm{wt} \%$ vs. $19 \mathrm{wt} \%$ ) but was also produced at a lower carbon yield (35\% vs. $40 \%)$. In spite of a four times higher B:C ratio during the FY19 experiments compared to FY18, the coke formation was similar or slightly lower (1.9 wt $\%$ vs. $2.1 \mathrm{wt} \%)$.

In addition to the CFP oil, $10.8 \%$ of the biomass carbon was present in light condensables. There were high carbon yields to three specific compounds: acetone $3.8 \%$, acetaldehyde $2.8 \%$, and MEK $1.2 \%$. The combined carbon yield for the CFP oil, acetone, acetaldehyde, and MEK was $43 \%$. The CFP oil contained only insignificant amounts of these three compounds. Additional metrics are shown in Table ES- 1.

\subsection{Area 300: CFP Product Condensation and Coproducts}

The process design for this section was maintained from the 2015 design report [1]. The system consists of two direct quench absorber/condensers. The upgraded vapors from the ex situ reactors are initially cooled via indirect heat exchange up to the modeled dew point of the vapor stream. A heavy organic liquid is then condensed in the first absorber/condenser; the light organic liquid product from the second condenser is used as the quench liquid. The uncondensed light vapors from the first condenser, as well as the vaporized quench liquid are then sent through heat exchangers. The partially condensed vapors enter the second absorber/condenser column for a final quench using a stream of recycled (and cooled) light organic liquid. The bottom product of the second condenser is separated into an aqueous waste stream (sent to Area 800) and an organic product. As previously mentioned, part of this light organic liquid product is also recycled for use as a quench liquid for both the absorber/condensers. In this design the heavy organic liquid from the first condenser and light organic liquid from the second condenser are mixed and sent to Area 400 for hydroprocessing.

A major addition to this section in the 2019 model was the recovery of light oxygenates from the gaseous stream of the second condenser. In the process design, the stream is sent to an adsorption system to remove the light oxygenates and other species, while letting the lighter gases pass through. The adsorbed species are then desorbed in a swing system. The desorbed stream is sent to a series of distillation columns to recover acetone and MEK as coproducts. The adsorption/desorption system cost was estimated from an ethanol dehydration mol-sieve system documented by Humbird et al. [22]. The scaling variable was moles of adsorbed species adjusted for partial pressure. The uptake of the desired species by the adsorbent is a key variable for determining the size of this system and model compound experiments showed that the uptake of acetone and MEK was in a similar range compared to the ethanol dehydration system.

Experiments will be conducted to determine the uptake of acetone and MEK from biomass CFP vapors (after the condensation of heavier species). 


\subsection{Area 400: CFP Oil Hydroprocessing}

The 2015 design report was written based on a premise that a single reactor system can handle the hydrotreating of the CFP organic liquid. Experiments in 2017-2019 have proven that this is a valid assumption and it is possible to get to less than $1 \%$ oxygen content in the CFP oil after a single hydrotreating step. Additional discussion was included in the 2018 SOT report [8]. For the 2019 SOT, a hydrotreating carbon efficiency of $95 \%$ was reported experimentally. The TEA model assumption was slightly lower to allow for some additional losses during hydrocracking of the heavier-than-diesel products; an overall hydrotreating + hydrocracking carbon efficiency of $93.5 \%$ was used in the model. The 2020 co-hydroprocessing TEA model maintains the same carbon efficiency assumptions for the CFP oil portion.

\subsection{Area 500: Hydrogen Production}

Hydrogen demands in the process were met (in the process models) without importing additional natural gas. Off-gases, primarily from CFP and other parts of the process, were processed in a steam reformer to produce hydrogen, and purified hydrogen was produced using pressure swing adsorption (PSA) units. Process design details for Area 500 are consistent with the 2015 design report [1]. The co-hydroprocessing case assumed the use of natural gas for hydrogen production at the petroleum refinery.

\subsection{Area 600: Steam System and Electricity Generation}

Heat available in the modeled process was used to generate electricity. Excess electricity, after meeting process demands, was sold to the grid. Process design details are consistent with the 2015 design report [1].

\subsection{Area 700: Cooling Water and Other Utilities}

Air cooling was the major cooling method in the process design when in-process heat recovery was not feasible. Process heat exchange and air-cooling costs are included with the costs of the respective process areas. Water cooling was used primarily for cooling process streams below $140^{\circ} \mathrm{F}$; process stream temperatures of $110^{\circ} \mathrm{F}$ were achieved after water cooling. Chilled water was used for cooling below $110^{\circ} \mathrm{F}$. Process design details for Area 700 are consistent with the 2015 design report [1].

\subsection{Area 800: Wastewater Utilization and Treatment}

Organic species in the wastewater stream were oxidized in a regenerative thermal oxidizer to allow discharge of the stream contents in an environmentally acceptable manner.

\subsection{Process Heat Exchange Cost}

A detailed heat exchange network was developed for the ex situ process and documented in the 2015 design report [1]. Cost estimates for the heat exchange networks in subsequent models were derived by scaling the costs from the design report using the total process heat exchange duty as the scaling basis. 


\section{Process Economics}

Capital and operating costs are listed in this section. Note that most of the information presented here is based on previously documented details $[1,6]$. Costs were updated to a 2016-dollars basis and scaled based on specific stream flows in the process models (as stated in Section 1.1.2).

Details for the 2019 SOT model and 2020 projection with the co-HP option are presented below. Information for the other models can be obtained from the tables in the Executive Summary and the Appendix.

\subsection{Total Capital Investment}

Installed capital costs are shown in Table 4, with per area total purchased equipment cost (TPEC), installation factors, and TIC.

Table 4. Total Installed Equipment Costs for the 2019 SOT and 2020 Projection with Co-HP

\begin{tabular}{|c|c|c|c|c|c|c|c|}
\hline \multirow[b]{2}{*}{ Area } & \multirow[b]{2}{*}{ Process Description } & \multicolumn{3}{|c|}{2019 SOT } & \multicolumn{3}{|c|}{2020 Co-HP Projection } \\
\hline & & $\begin{array}{l}\text { TPEC } \\
\text { (MM\$) }\end{array}$ & $\mathbf{f}_{\text {install }}$ & $\begin{array}{r}\text { TIC } \\
(\mathrm{MM} \$)\end{array}$ & $\begin{array}{l}\text { TPEC } \\
\text { (MM\$) }\end{array}$ & $\mathbf{f}_{\text {install }}$ & $\begin{array}{r}\text { TIC } \\
\text { (MM\$) }\end{array}$ \\
\hline 100 & Feed handling and drying ${ }^{a}$ & 0.3 & 1.96 & 0.5 & 0.3 & 1.96 & 0.6 \\
\hline 200 & $\begin{array}{l}\text { Fast pyrolysis and vapor } \\
\text { upgrading }\end{array}$ & 44.8 & 2.52 & 112.7 & 44.7 & 2.52 & 112.4 \\
\hline 300 & $\begin{array}{l}\text { Pyrolysis vapor quench, } \\
\text { condensation and coproduct } \\
\text { recovery }\end{array}$ & 21.0 & 1.84 & 38.7 & 24.9 & 1.84 & 45.8 \\
\hline 400 & $\begin{array}{l}\text { Hydroprocessing and product } \\
\text { separation }\end{array}$ & 16.5 & 1.77 & 29.2 & $0^{b}$ & - & $0 \mathrm{~b}$ \\
\hline 500 & Hydrogen plant & 35.4 & 1.95 & 69.3 & 24.8 & 2.03 & 50.1 \\
\hline 600 & $\begin{array}{l}\text { Steam system and power } \\
\text { generation }\end{array}$ & 25.7 & 1.84 & 47.3 & 33.0 & 1.84 & 60.8 \\
\hline 700 & Cooling water and other utilities & 4.3 & 2.02 & 8.8 & 4.4 & 2.02 & 8.9 \\
\hline 800 & $\begin{array}{l}\text { Wastewater management and } \\
\text { recycle }\end{array}$ & 10.3 & 2.35 & 24.2 & 10.1 & 2.34 & 23.6 \\
\hline \multicolumn{2}{|c|}{ ISBL (Areas 100-400) } & 82.5 & 2.19 & 181.2 & 69.9 & 2.27 & 158.8 \\
\hline \multicolumn{2}{|c|}{ OSBL (Areas 500-800) } & 75.8 & 1.97 & 149.6 & 72.3 & 1.98 & 143.5 \\
\hline \multicolumn{2}{|l|}{ Total } & 158.4 & 2.09 & 330.8 & 142.2 & 2.13 & 302.3 \\
\hline
\end{tabular}

a Most investment costs for feed handling and drying are included in the per-unit woody feedstock price. This cost is for a secondary biomass dryer that serves to recover heat. ISBL = inside battery limits; OSBL = outside battery limits. ${ }^{b}$ Capital at the biorefinery is zero because co-hydroprocessing occurs at the petroleum refinery in this model.

The sum of equipment purchases and installation/construction costs is defined as the total direct cost (TDC). Indirect costs, such as project management and engineering, procurement, and construction services, are estimated with factors on the TDC as shown in Table 5. 
Table 5. Cost Factors for Indirect Costs

\begin{tabular}{lr}
\hline Indirect Costs & \% of TDC \\
\hline Prorated expenses & 10.0 \\
Home office and construction fees & 20.0 \\
Field expenses & 10.0 \\
Project contingency & 10.0 \\
Other costs (startup and permits) & 10.0 \\
\hline Total Indirect Costs & $\mathbf{6 0 . 0}$ \\
\hline
\end{tabular}

* Excluding land purchase cost.

The sum of direct and indirect costs is defined as the fixed capital investment (FCI). The working capital is estimated to be $5 \%$ of the FCI. The sum of FCI and working capital is the TCI. Table 6 presents a summary of these capital quantities for the 2019 SOT and 2020 co-HP projection.

Table 6. Total Capital Investment Calculations

\begin{tabular}{|c|c|c|c|}
\hline & & 2019 SOT & $\begin{array}{l}2020 \text { Co-HP } \\
\text { Projection }\end{array}$ \\
\hline $\begin{array}{l}\text { Total purchased equipment cost } \\
\text { (TPEC) }\end{array}$ & & $\$ 158,380,000$ & $\$ 142,210,000$ \\
\hline Installation factor & & 2.088 & 2.126 \\
\hline Total installed cost (TIC) & & $\$ 330,770,000$ & $\$ 302,330,000$ \\
\hline \multicolumn{4}{|l|}{ Other direct costs } \\
\hline Land (not depreciated) & & $\$ 1,610,000$ & $\$ 1,610,000$ \\
\hline Warehouse & $4.0 \%$ of ISBL & $\$ 7,250,000$ & $\$ 6,350,000$ \\
\hline Site development & $10.0 \%$ of ISBL & $\$ 18,120,000$ & $\$ 15,880,000$ \\
\hline Additional piping & $4.5 \%$ of ISBL & $\$ 8,150,000$ & $\$ 7,150,000$ \\
\hline Total direct costs (TDC) & & $\$ 364,290,000$ & $\$ 331,710,000$ \\
\hline Indirect costs & $\%$ of TDC (ex land) & & \\
\hline Prorated expenses & $10.0 \%$ & $\$ 36,430,000$ & $\$ 33,170,000$ \\
\hline Home office and construction fees & $20.0 \%$ & $\$ 72,860,000$ & $\$ 66,340,000$ \\
\hline Field expenses & $10.0 \%$ & $\$ 36,430,000$ & $\$ 33,170,000$ \\
\hline Project contingency & $10.0 \%$ & $\$ 36,430,000$ & $\$ 33,170,000$ \\
\hline Other costs (startup and permits) & $10.0 \%$ & $\$ 36,430,000$ & $\$ 33,170,000$ \\
\hline Total indirect costs & $60.0 \%$ & $\$ 218,570,000$ & $\$ 199,030,000$ \\
\hline Fixed capital investment (FCI) & & $\$ 582,860,000$ & $\$ 530,740,000$ \\
\hline Working capital & $5.0 \%$ of $\mathrm{FCl}$ (ex land) & $\$ 29,140,000$ & $\$ 26,540,000$ \\
\hline Total capital investment (TCI) & & $\$ 612,000,000$ & $\$ 557,280,000$ \\
\hline TCI/TPEC & & 3.864 & 3.919 \\
\hline $\mathrm{FCI}$ Lang Factor $=\mathrm{FCl} / \mathrm{ISBL}$ TPEC & & 7.060 & 7.592 \\
\hline $\mathrm{TCI}$ Lang Factor $=\mathrm{TCI} / \mathrm{ISBL}$ TPEC & & 7.413 & 7.972 \\
\hline
\end{tabular}




\subsection{Operating Costs}

Variable operating cost assumptions are shown in Table 7. The major addition to the table in the 2015 design report [1] is the $\mathrm{Pt} / \mathrm{TiO}_{2}$ catalyst used in the fixed-bed ex situ reactor.

Table 7. Variable Operating Cost Assumptions

\begin{tabular}{|c|c|}
\hline Variable & Information and Operating Cost (cost year in parentheses) \\
\hline \multirow{2}{*}{$\begin{array}{l}\text { Fluidized bed } \\
\text { media }\end{array}$} & For fast pyrolysis, the bed medium is sand. \\
\hline & Sand price: $\$ 45.74 /$ U.S. ton $(2011 \$)$ [17]; initial fill, then make up for attrition. \\
\hline $\begin{array}{l}\text { Fixed-bed ex situ } \\
\text { vapor upgrading } \\
\text { catalyst }\end{array}$ & $\begin{array}{l}\mathrm{Pt} / \mathrm{TiO}{ }_{2} \text { with } 0.5 \% \mathrm{Pt} \text { loading. Catalyst unit cost } \$ 92.35 / \mathrm{lb}(2014 \$)[15] \text {. A } 2 \text {-year } \\
\text { lifetime is assumed, with } 70 \% \text { cost recovery at the end of } 2 \text { years. Quantity of } \\
\text { catalyst was determined using a weight hourly space velocity (WHSV) of } 5 \mathrm{~h}^{-1} \\
\text { based on vapor flow and an additional } 70 \% \text { overdesign. }\end{array}$ \\
\hline \multirow[t]{2}{*}{$\begin{array}{l}\text { Hydrotreating and } \\
\text { hydrocracking } \\
\text { catalysts }\end{array}$} & $\begin{array}{l}\text { To determine the amount of catalyst inventory, the hydroprocessors were sized for } \\
\text { a WHSV of } 0.5 \mathrm{~h}^{-1} \text { based on the expected hydroprocessing severity. Initial fill is } \\
\text { then replaced every } 2 \text { years. }\end{array}$ \\
\hline & $\begin{array}{l}\text { Price: } \$ 20 / \mathrm{lb}(2011 \$) \text { based on NREL calculations using metals pricing and costs } \\
\text { for manufacturing processes and some buffer for modifications. }\end{array}$ \\
\hline \multirow{2}{*}{$\begin{array}{l}\text { Steam methane } \\
\text { reformer catalysts }\end{array}$} & Based on a literature value of price per unit hydrogen produced. \\
\hline & Price: $\$ 7.80 /$ U.S. ton hydrogen $(2011 \$)[18]$. \\
\hline \multirow[t]{3}{*}{ Natural gas } & $\begin{array}{l}\text { Purchased from pipeline for feed to steam methane reformer for hydrogen } \\
\text { production. Natural gas has an insignificant cost contribution because of minimal } \\
\text { process use. }\end{array}$ \\
\hline & Price at biorefinery: $\$ 239 /$ U.S. ton (\$5/MMBtu) (2011\$). \\
\hline & $\begin{array}{l}\text { For the co-hydroprocessing case, natural gas was } \$ 3.5 / \mathrm{MMBtu} \text {, based on a } 10- \\
\text { year average for Texas using EIA information (https://www.eia.gov/naturalgas/). }\end{array}$ \\
\hline Solids disposal & Price: $\$ 33 /$ tonne (1998\$) [19]. \\
\hline \multirow[t]{2}{*}{ Diesel fuel } & Usage: $10 \mathrm{gal} / \mathrm{h}$ plant-wide use. \\
\hline & $\begin{array}{l}2012 \text { price projection: } \$ 21.29 / \mathrm{MMBtu}(2009 \$)[20]=\$ 2.86 / \text { gal at } 0.85 \text { specific } \\
\text { gravity. }\end{array}$ \\
\hline Water makeup & Price: $\$ 0.22 /$ tonne $(2001 \$)[21]=\$ 0.20 /$ U.S. ton. \\
\hline \multirow[t]{3}{*}{ Chemicals } & Boiler feedwater chemicals_-Price: \$75/MM Ib blowdown (2014 vendor estimate). \\
\hline & $\begin{array}{l}\text { Cooling tower chemicals-Price: } \$ 33.84 / y r \text { per U.S. ton of cooling capacity (2014 } \\
\text { vendor estimate). }\end{array}$ \\
\hline & Caustic-Price: $\$ 150 /$ dry U.S. ton (2010\$) [22]. \\
\hline \multirow[t]{2}{*}{ Wastewater } & $\begin{array}{l}\text { Most wastewater is cleaned using a reverse osmosis system and recycled. } \\
\text { Additional treatment is assumed for the balance. }\end{array}$ \\
\hline & Price: $\$ 0.022 /$ gallon (2011\$). Based on Humbird et al. [22]. \\
\hline
\end{tabular}

Note: Costs shown were updated to 2016 dollars using the Producer Price Index for chemical manufacturing [10]. 
Fixed operating costs related to salaries are shown in Table 8. Number of personnel and their salaries were maintained from the 2015 design report [1], and salaries were adjusted using a labor cost index [23].

Table 8. Fixed Operating Costs

\begin{tabular}{lrrr}
\hline Cost Item & Factor & 2019 SOT & $\begin{array}{c}\text { 2020 Co-HP } \\
\text { Projection }\end{array}$ \\
\hline Salaries & & $\$ 3,440,000$ & $\$ 3,440,000$ \\
Benefits and overhead & $90.0 \%$ of total salaries (after adding $10 \%)$ & $\$ 3,100,000$ & $\$ 3,100,000$ \\
Maintenance & $3.0 \%$ of fixed capital investment $\left(\mathrm{FCl}^{\star}\right)$ & $\$ 17,486,000$ & $\$ 15,922,000$ \\
Insurance and taxes & $0.7 \%$ of fixed capital investment $\left(\mathrm{FCl}^{*}\right)$ & $\$ 4,080,000$ & $\$ 3,715,000$ \\
\hline Total fixed operating costs (2016\$/year) & $\mathbf{\$ 2 8 , 0 9 2 , 0 0 0}$ & $\mathbf{\$ 2 6 , 1 6 4 , 0 0 0}$ \\
\hline
\end{tabular}

* Percentages of $\mathrm{FCl}$ exclude land purchase cost.

\subsection{Discounted Cash Flow Analysis and the Minimum Fuel Selling Price}

Once the capital and operating costs are determined, the GGE of fuel production is used to calculate an MFSP (in \$/GGE) using a discounted cash flow rate of return (DCFROR) analysis. Further details are available in the 2015 design report [1].

\subsection{Value of Hydrocarbon Fuel Products}

The results of the cash flow analyses for the 2019 SOT and the 2020 co-HP projection are summarized in Table 9. Gasoline and diesel are normalized by lower heating value to represent a single gasoline-equivalent product and MFSP. To calculate individual selling prices for gasoline and diesel, the MFSP per GGE is ratioed back to these products by lower heating value. The densities of the blendstocks were assumed to be those of U.S. conventional gasoline $(2,819$ grams/gallon or $6.215 \mathrm{lb} /$ gallon) and diesel fuels (3,167 grams/gallon or $6.982 \mathrm{lb} /$ gallon) [24].

Table 9. Projected Selling Prices of Hydrocarbon Blendstocks

\begin{tabular}{|c|c|c|}
\hline & 2019 SOT & $\begin{array}{r}2020 \text { Co-HP } \\
\text { Projection }\end{array}$ \\
\hline $\begin{array}{l}\text { Lower heating value for gasoline-range products } \\
\text { (simulation result) }\end{array}$ & $111,862 \mathrm{Btu} / \mathrm{gal}$ & $111,882 \mathrm{Btu} / \mathrm{gal}$ \\
\hline $\begin{array}{l}\text { Lower heating value for diesel-range products } \\
\text { (simulation result) }\end{array}$ & $126,739 \mathrm{Btu} / \mathrm{gal}$ & $126,739 \mathrm{Btu} / \mathrm{gal}$ \\
\hline Calculated gasoline-equivalent MFSP & $\$ 3.33 /$ GGE & $\$ 3.09 /$ GGE \\
\hline Calculated actual MFSP for gasoline-range products & $\$ 3.21 / \mathrm{gal}$ & $\$ 2.98 / \mathrm{gal}$ \\
\hline Calculated actual MFSP for diesel-range products & $\$ 3.63 / \mathrm{gal}$ & $\$ 3.37 /$ gal \\
\hline Gasoline lower heating value for GGE normalization & & $116,090 \mathrm{Btu} / \mathrm{gal}$ \\
\hline
\end{tabular}




\section{Process Economics Summary}

The contribution of individual areas toward the MFSP on a $\$$ GGE basis are shown in Figure 2 (2019 SOT) and Figure 3 (2020 projection for co-HP option).

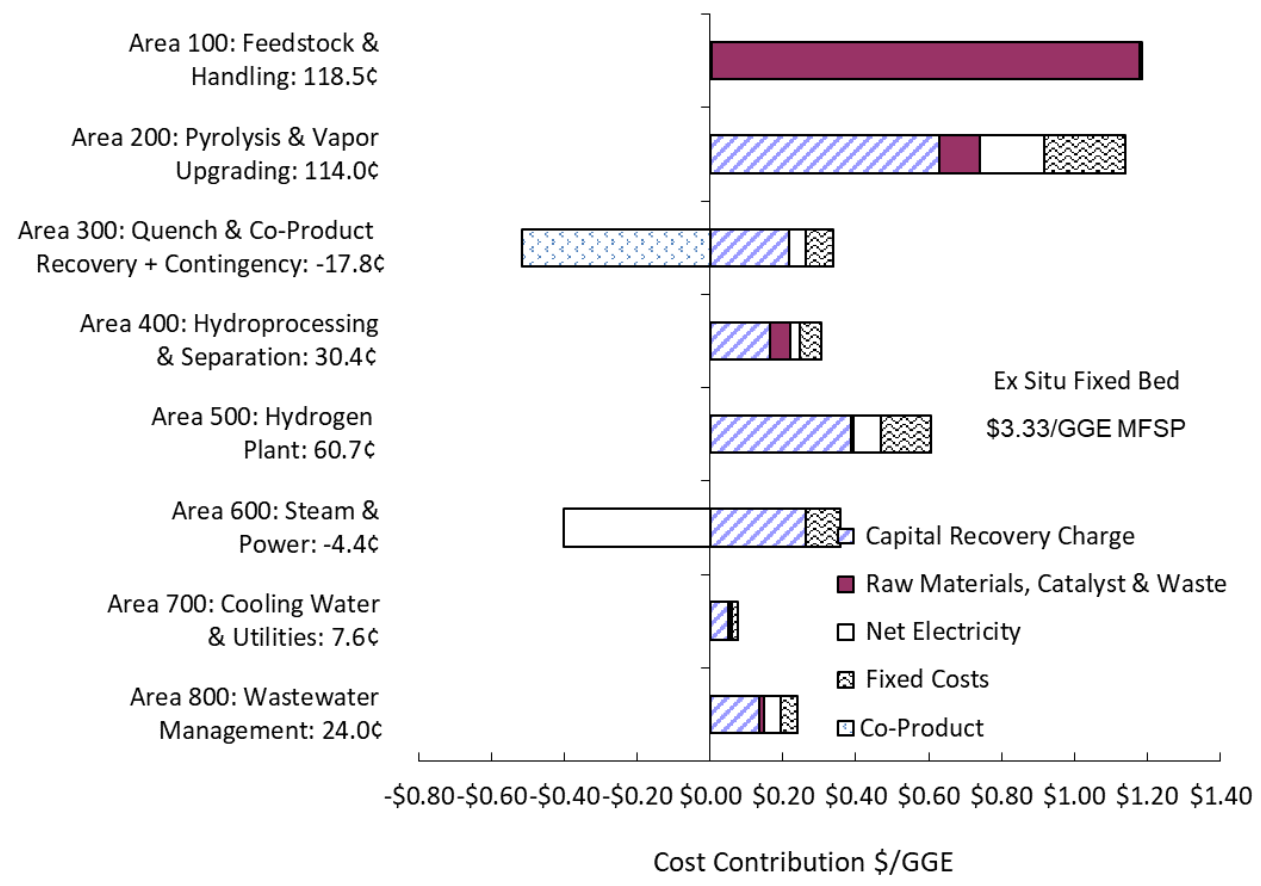

Figure 2. Cost contribution details from each process area for the 2019 SOT

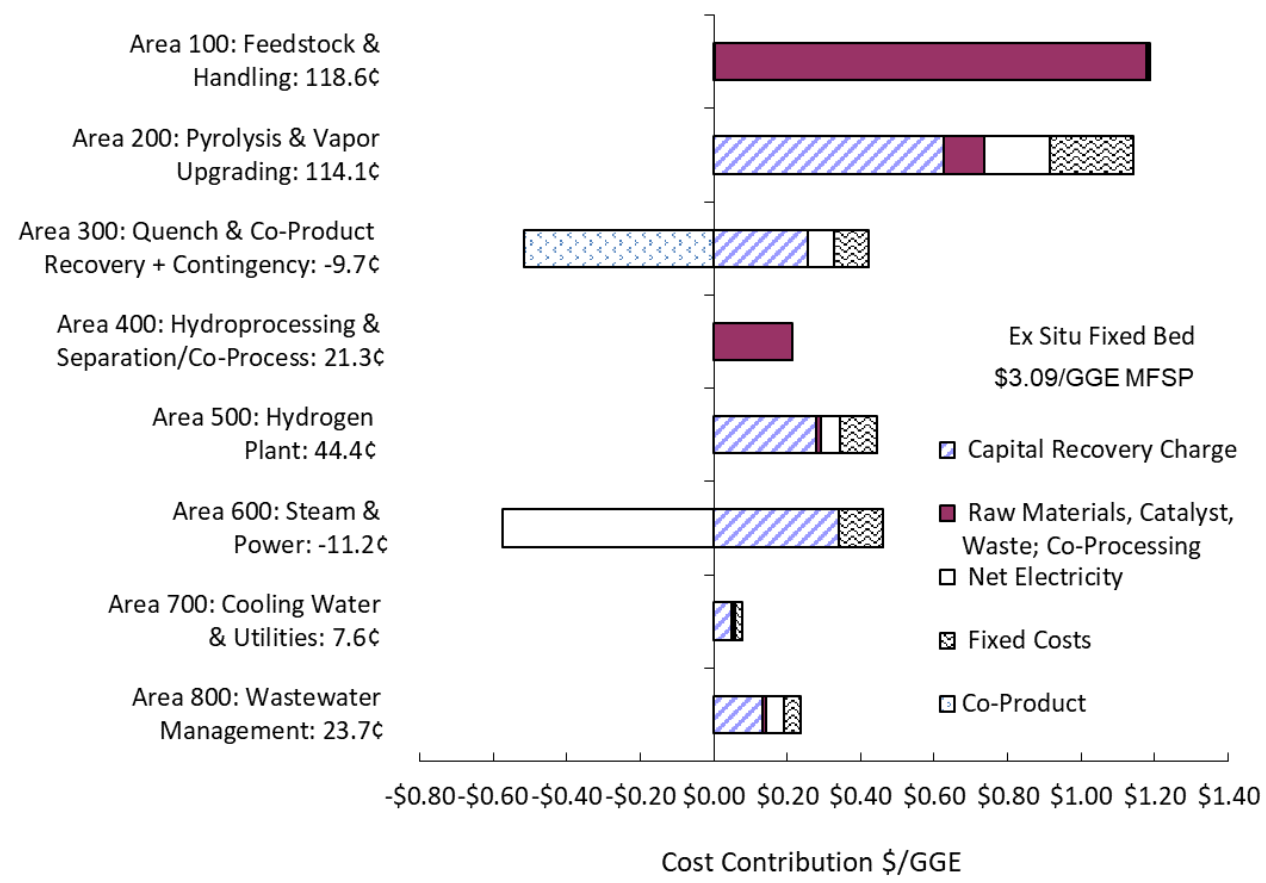

Figure 3. Cost contribution details from each process area for the 2020 co-HP projection 
Note the biggest changes in the 2020 co-HP projection case presented above compared to the 2019 SOT case: (1) savings from lower hydroprocessing and hydrogen costs; (2) additional electricity generation at the biorefinery because of the availability of off-gases otherwise used for additional hydrogen production; and (3) additional contingency at the biorefinery (lumped in Area 300) related to any changes in configuration to allow co-hydroprocessing. 


\section{Sustainability and Life Cycle Analysis}

This section presents sustainability metric indicators for the 2019 SOT and 2020 co-HP projection conversion process models. Direct air emissions from the biorefinery $\left(\mathrm{CO}_{2}, \mathrm{NO}_{2}\right.$, and $\mathrm{SO}_{2}$ ), water consumption, and other process-related metrics were taken from the Aspen Plus models. The material and energy flow information from the conversion models also help capture the impacts of input raw materials, and outputs such as fuel yields, waste, and coproducts. Pertinent flows are shown in Table 10.

The input/output inventories in Table 10 also provide the necessary information required for performing life cycle and supply chain sustainability modeling to quantify greenhouse gas (GHG) emissions and fossil energy consumption. The biorefinery GHGs and fossil energy consumption are quantified separately under supply chain sustainability analysis (SCSA) efforts by Argonne National Laboratory (ANL). A complete supply chain life cycle analysis (LCA) is required to understand the sustainability implications of this technology pathway and quantify associated reduction in GHG emissions from the production of the biomass-derived liquid fuel blendstock (compared to petroleum-derived liquid fuels). 
Table 10. Material and Energy Flows in the Conversion Process

\begin{tabular}{|c|c|c|}
\hline & 2019 SOT & $\begin{array}{r}2020 \text { Co-HP } \\
\text { Projection }\end{array}$ \\
\hline Products & $\mathrm{lb} / \mathrm{h}$ & $\mathrm{lb} / \mathrm{h}$ \\
\hline Gasoline Fuel & 18,189 & 18,189 \\
\hline Diesel Fuel & 16,913 & 16,913 \\
\hline \multicolumn{3}{|l|}{ Byproducts } \\
\hline Excess Electricity (kW) & 1997 & 20031 \\
\hline MEK (Ib/h) & 1178 & 1178 \\
\hline Acetone $(\mathrm{lb} / \mathrm{h})$ & 5046 & 5046 \\
\hline Resource Consumption at Petroleum Refinery & Flow rate & Flow rate \\
\hline Fuel Natural Gas (MMBtu/h) & - & 239 \\
\hline Electricity (kW) & - & 2719 \\
\hline Steam (lb/h) & - & -4507 \\
\hline Cooling Water Makeup (gal/h) & - & 6014 \\
\hline Hydrotreating Catalyst (lb/h) & - & 14 \\
\hline Hydrocracking Catalyst (lb/h) & - & 2 \\
\hline \multirow[t]{2}{*}{ Resource Consumption at Biorefinery } & Flow rate & Flow rate \\
\hline & $\mathrm{lb} / \mathrm{h}$ & $\mathrm{lb} / \mathrm{h}$ \\
\hline Blended Woody Biomass (wet) & 204,131 & 204,131 \\
\hline Blended Woody Biomass (dry) & 183,718 & 183,718 \\
\hline Sand Makeup & 158 & 158 \\
\hline Natural Gas & 48 & 357 \\
\hline Zeolite Catalyst & $0.0 \mathrm{E}+00$ & $0.0 \mathrm{E}+00$ \\
\hline Fixed-Bed VPU Catalyst $\left(\mathrm{Pt} / \mathrm{TiO}_{2}\right)$ & 7 & 7 \\
\hline Hydrotreating Catalyst (sulfided CoMo or NiMo) & 14 & - \\
\hline Hydrocracking Cat. (crystalline Si-Al with rare earth metals) & 2 & - \\
\hline $\mathrm{ZnO}$ (reforming cleanup) & $1.2 \mathrm{E}-02$ & 9.1E-02 \\
\hline HDS (reforming cleanup) & $5.2 \mathrm{E}-03$ & $3.9 \mathrm{E}-02$ \\
\hline Steam Reforming Catalyst & 2.7E-02 & 2.0E-01 \\
\hline Shift Catalyst & 3.7E-02 & 2.7E-01 \\
\hline PSA Adsorbent & $9.2 \mathrm{E}-01$ & $6.9 \mathrm{E}+00$ \\
\hline 50 wt\% Caustic & 291 & 291 \\
\hline Net Water Makeup & 75,835 & 53,545 \\
\hline Boiler Feedwater Chemicals & 2 & 2 \\
\hline Cooling Tower Chemicals & 1 & 1 \\
\hline No. 2 Diesel Fuel & 71 & 71 \\
\hline Waste Streams & $\mathrm{lb} / \mathrm{h}$ & $\mathrm{lb} / \mathrm{h}$ \\
\hline Solids Purge from Fluidized Bed Reactors & 3,741 & 3,741 \\
\hline
\end{tabular}




\begin{tabular}{lrr} 
Wastewater & 25,189 & 22,147 \\
\hline Air Emissions & $\mathrm{lb} / \mathrm{h}$ & $\mathrm{lb} / \mathrm{h}$ \\
\hline $\mathrm{CO}_{2}$ (fossil) & 132 & 980 \\
$\mathrm{CO}_{2}$ (biogenic) & 196,717 & 197,545 \\
$\mathrm{CH}_{4}$ & $0.0 \mathrm{E}+00$ & $0.0 \mathrm{E}+00$ \\
$\mathrm{CO}$ & $0.0 \mathrm{E}+00$ & $0.0 \mathrm{E}+00$ \\
$\mathrm{NO}_{2}$ & 9 & 11 \\
$\mathrm{SO}_{2}$ & 106 & 106 \\
$\mathrm{H}_{2} \mathrm{O}$ & 135,165 & 150,488 \\
$\mathrm{H}_{2} \mathrm{~S}$ & $0.0 \mathrm{E}+00$ & $0.0 \mathrm{E}+00$ \\
\hline Combustor Feed Stream Heating Values & & \\
\hline Char Combustor \% Biogenic Carbon & $100 \%$ & $100 \%$ \\
Char Combustor LHV (MMBtu/h) & 295.94 & 295.94 \\
Reformer Fuel Combustor \% Biogenic Carbon & $99.89 \%$ & $99.17 \%$ \\
Reformer Fuel Combustor LHV (MMBtu/h) & 305.43 & 458.66 \\
\hline
\end{tabular}

Table 11 shows efficiency indicators and water usage metrics in the conversion process. Carbon and energy efficiencies also reflect the sustainability of the process; conversion of biomass feedstock to desirable products benefits both the economics and sustainability. The process does not require natural gas at the biorefinery, and the model predicts that there will be a net electricity export after in-process consumption (the electricity is produced from excess process heat); however, natural gas and electricity are consumed at the petroleum refinery for the co-HP case, as shown in Table 10.

Table 11. Sustainability and Process Efficiency Metrics for the Conversion Process

\begin{tabular}{lcccc}
\hline Model & \multicolumn{2}{c}{ 2019 SOT } & \multicolumn{2}{c}{$\begin{array}{c}\text { 2020 Co-HP } \\
\text { Projection }\end{array}$} \\
\cline { 2 - 5 } Electricity \& Coproduct Credit & Yes & No & Yes & No \\
\hline GHG Emissions (g CO ${ }_{2}$ e/MJ) ${ }^{\text {a }}$ & -19.8 & 1.0 & $-5.1^{\mathrm{b}}$ & $29.4 \mathrm{~b}$ \\
Net Fossil Energy Consumption (MJ/MJ) ${ }^{\mathrm{b}}$ & -0.26 & 0.01 & 0.02 & 0.45 \\
\hline Fuel Yield (\% w/w of dry biomass) & 19.1 & 19.1 \\
Carbon Efficiency to Fuels (\% of C in biomass) & 33.0 & 32.9 \\
Overall Energy Efficiency to Fuels (\% LHV basis) & 43.6 & 43.4 \\
Water Consumption (gal/GGE fuel) & 1.7 & $1.2 \mathrm{c}$ \\
Total Fuel Yield (GGE/dry U.S. ton) & 59.5 & 59.5 \\
Electricity Production (kWh/GGE) & 7.8 & 10.8 \\
Electricity Consumption (kWh/GGE) & 7.4 & 7.1 \\
Wastewater Generation (gal/GGE) & \multicolumn{2}{c}{0.55} & 0.49 \\
\hline a Calculated by ANL using Greenhouse gases, Regulated Emissions, and Energy use in Transportation v. \\
2018 [25] at the conversion step (i.e., at the biorefinery or "gate-to-gate"), excluding upstream and \\
downstream processes in the supply chain. The full SCSA results will be reported separately by ANL. \\
bIncludes emissions at petroleum refinery. ${ }^{\mathrm{c}}$ At biorefinery.
\end{tabular}


An SCSA for the ex situ CFP pathway was conducted using ANL's Greenhouse gases, Regulated Emissions, and Energy use in Transportation or GREET model [25]. The SCSA incorporated the feedstock modeled by INL for the 2019 SOT (50\% forest residues and 50\% clean pine) [13]. For the conversion step, when the displacement credit of coproduced electricity is excluded, fossil energy consumption is approximately 0.01 and $0.45 \mathrm{MJ} / \mathrm{MJ}$ for the 2019 SOT and 2020 co-HP projection, respectively; GHG emission intensities are approximately 1.0 and $29.4 \mathrm{~g} \mathrm{CO}_{2} \mathrm{e} / \mathrm{MJ}$, respectively, for the 2019 SOT and 2020 co-HP projection. When the displacement credit of coproduced electricity and coproducts is included, net fossil energy consumption is approximately -0.26 and $0.02 \mathrm{MJ} / \mathrm{MJ}$ for the 2019 SOT and 2020 co-HP projection, respectively; net GHG emission intensities are approximately -19.8 and $-5.1 \mathrm{~g} \mathrm{CO}_{2} \mathrm{e} / \mathrm{MJ}$ for the $2019 \mathrm{SOT}$ and 2020 co-HP projection cases, respectively. Energy self-sufficient conversion processes contribute to the low fossil energy consumption and low GHG emission intensities of the ex situ CFP conversion technology at the biorefinery. The acetone and MEK coproducts provide further GHG benefits based on the LCA product displacement method [26]; however, there is natural gas and grid electricity consumption at the petroleum refinery that increase GHG emissions for the production of final fuel for the $2020 \mathrm{co}-\mathrm{HP}$ case. It should be noted that the hydrogen consumption estimates at the petroleum refinery will be revisited; this report provides initial estimates.

ANL's SCSA shows that the overall modeled GHG reduction relative to a petroleum-derived gasoline baseline ( $95.3 \mathrm{gCO} e / \mathrm{MJ}$ ) is greater than $60 \%$ for the 2019 SOT (forthcoming publication [27]). ANL's calculations also quantified a 52\% reduction for the $2020 \mathrm{co}-\mathrm{HP}$ projection. Feedstock choices and related preprocessing can have significant impacts on the SCSA results and can thus be an area of improvement in this respect. Co-hydroprocessing at a petroleum refinery using an assumption of hydrogen production from natural gas also impacts the GHG reduction for the 2020 co-HP case. 


\section{Conclusions and Future Work}

There were significant updates to this conversion pathway since the 2018 SOT report. These include the accounting of nearly $100 \%$ of the biomass carbon in the CFP products; as a result of this quantification, it was found that there are significantly more light oxygenates in the products than previously quantified. Acetaldehyde, acetone, and MEK constitute a significant proportion of those light oxygenates. This necessitated the investigation of ways to recover, purify, and derive value from the light oxygenates by selling acetone and MEK as coproducts. Model compound experiments allowed the selection of a suitable adsorbent to capture these key coproducts and experiments will be conducted using biomass CFP vapors in the near future; this will help validate adsorption performance and related TEA assumptions. Other significant achievements include the successful use of low-cost feedstocks (that included forest residues) during bench scale experiments and a significant extension of onstream times of the fixed-bed ex situ CFP catalyst. Stand-alone hydroprocessing experiments for CFP oil have been successful so far with respect to oxygen content reduction in a single hydrotreating step. Co-hydroprocessing of CFP oil with petroleum refinery streams is being considered as an option to help reduce costs and enhance commercial relevance of this conversion pathway. Pilot-scale verification of key parts of this technology is expected in 2022; a 500-hour bench scale experimental campaign will be conducted in 2020 to prove the feasibility of scaling up this technology. 


\section{References}

1. Dutta, A., A. Sahir, E. Tan, D. Humbird, L. Snowden-Swan, P. Meyer, J. Ross, D. Sexton, R. Yap, and J. Lukas. 2015. Process Design and Economics for the Conversion of Lignocellulosic Biomass to Hydrocarbon Fuels-Thermochemical Research Pathways with in situ and ex situ Upgrading of Fast Pyrolysis Vapors. NREL/TP-5100-62455, PNNL-23823. Golden, CO: National Renewable Energy Laboratory.

2. U.S. Department of Energy. 2016. Bioenergy Technologies Office Multi-Year Program Plan. Washington, DC: U.S. Department of Energy.

3. Lappas, A.A., M.C. Samolada, D.K. Iatridis, S.S. Voutetakis, and I.A. Vasalos. 2002. "Biomass pyrolysis in a circulating fluid bed reactor for the production of fuels and chemicals." Fuel 81, no. 16: 2087-2095. https://doi.org/10.1016/S0016-2361(02)00195$\underline{3}$.

4. Wan, S., T. Pham, S. Zhang, L. Lobban, D. Resasco, and R. Mallinson. 2013. "Direct catalytic upgrading of biomass pyrolysis vapors by a dual function $\mathrm{Ru} / \mathrm{TiO}_{2}$ catalyst." AIChE Journal 59, no. 7: 2275-2285. https://doi.org/10.1002/aic.14038.

5. Wang, K., D.C. Dayton, J.E. Peters, and O.D. Mante. 2017. "Reactive catalytic fast pyrolysis of biomass to produce high-quality bio-crude." Green Chem 19: 243-3251. https://doi.org/10.1039/C7GC01088E.

6. Dutta, A., J. Schaidle, D. Humbird, F. Baddour, and A. Sahir. 2016. "Conceptual Process Design and Techno-Economic Assessment of Ex Situ Catalytic Fast Pyrolysis of Biomass: A Fixed Bed Reactor Implementation Scenario for Future Feasibility.” Topics in Catalysis 59, no. 1: 2-18. https://doi.org/10.1007/s11244-015-0500-z.

7. Griffin, M.B., K. Iisa, H. Wang, A. Dutta, K.A. Orton, R.J. French, and D.M. Santosa et al. 2018. "Driving towards cost-competitive biofuels through catalytic fast pyrolysis by rethinking catalyst selection and reactor configuration." Energy Environ Sci 11: 29042918. http://dx.doi.org/10.1039/C8EE01872C.

8. Dutta, A., K. Iisa, C. Mukarakate, M. Griffin, E.C.D. Tan, J. Schaidle, D. Humbird, H. Wang, D. Hartley, D. Thompson, and H. Cai. 2018. Ex Situ Catalytic Fast Pyrolysis of Lignocellulosic Biomass to Hydrocarbon Fuels: 2018 State of Technology and Future Research. NREL/TP-5100-71954. Golden, CO: National Renewable Energy Laboratory.

9. Internal Revenue Service. 2009. "How to Depreciate Property.” Internal Revenue Service Publication 946. Washington, DC: United States Department of the Treasury.

10. U.S. Bureau of Labor Statistics. 2018. "PPI industry sub-sector data for Chemical Manufacturing." Accessed October 26, 2018. https://data.bls.gov/timeseries/PCU325--325---?amp\%253bdata_tool=XGtable\&output_view=data\&include graphs=true.

11. Chemical Engineering. 2018. "Chemical Engineering Plant Cost Index.” Accessed September 2018. 
12. Aspen Plus. n.d. Bedford, MA: AspenTech. https://www.aspentech.com.

13. Hartley, Damon S., David N. Thompson, Hongqiang Hu, and Hao Cai. 2018. Woody Feedstock 2018 State of Technology Report. INL/EXT-18-51655. Idaho Falls, ID: Idaho National Laboratory.

14. Hu, Hongqiang, Tyler L. Westover, Robert Cherry, John E. Aston, Jeffrey A. Lacey, and David N. Thompson. 2017. "Process simulation and cost analysis for removing inorganics from wood chips using combined mechanical and chemical preprocessing." BioEnergy Research 10, no. 1: 237-247. https://doi.org/10.1007/s12155-016-9794-3.

15. Baddour, F., and L. Snowden-Swan. 2017. "Catalyst Cost Model Development." Presented at Bioenergy Technologies Office 2017 Project Peer Review, Denver, CO, March 7, 2017. https://www.energy.gov/sites/prod/files/2017/05/f34/thermochem baddour 2.5.4.301302.pdf.

16. Chemical Catalysis for Bioenergy Consortium. n.d. https://www.chemcatbio.org.

17. "Sand and Gravel (Industrial)." 2014. U.S. Geological Survey. Accessed August 12, 2014. http://minerals.usgs.gov/minerals/pubs/commodity/silica/mcs-2014-sandi.pdf.

18. Suresh, B., R. Gubler, Y. Yamaguchi, and X. He. 2013. "Hydrogen.” In Chemical Economics Handbook. Englewood, CO: IHS Chemical.

19. Peters, M.S., K.D. Timmerhaus, and R. West. 2004. Plant Design and Economics for Chemical Engineers, International Edition. 5th edition. New York: McGraw-Hill, Inc.

20. U.S. Energy Information Administration. 2011. "Annual Energy Outlook 2011. Data Table A3-Energy Prices by Sector and Source." Washington, DC: U.S. Energy Information Administration.

21. Perry, R.H., D.W. Green, and J.O. Maloney. 1997. Perry's Chemical Engineers' Handbook. 7th edition. New York: McGraw-Hill.

22. Humbird, D., R. Davis, L. Tao, C. Kinchin, D. Hsu, A. Aden, P. Schoen, et al. 2011. Process Design and Economics for Biochemical Conversion of Lignocellulosic Biomass to Ethanol: Dilute-Acid Pretreatment and Enzymatic Hydrolysis of Corn Stover. NREL/TP-5100-47764. Golden, CO: National Renewable Energy Laboratory.

23. U.S. Bureau of Labor Statistics. 2018. "Databases, Tables \& Calculators by Subject." Accessed 2018: http://data.bls.gov/cgi-bin/srgate (enter CEU3232500008 series id).

24. Argonne National Laboratory. 2014. "GREET 2016 Model." Accessed 2018: https://greet.es.anl.gov/publication-greet-model. 
25. Wang, Michael, Amgad Elgowainy, Pahola Thathiana Benavides, Andrew Burnham, Hao Cai, Qiang Dai, Troy Robert Hawkins et al. 2018. Summary of Expansions and Updates in GREET 2018. ANL-18-38. Lemont, IL: Argonne National Laboratory.

26. Cai, H., J. Han, M. Wang, R. Davis, M. Biddy, E. Tan. 2018. "Life-cycle analysis of integrated biorefineries with co-production of biofuels and bio-based chemicals: coproduct handling methods and implications." Biofpr 12: 815-833. http://dx.doi.org/10.1039/C8EE01872C

27. H. Cai, L. Ou, M. Wang, E. Tan, R. Davis, A. Dutta et al. 2020. Supply Chain Sustainability Analysis of Renewable Hydrocarbon Fuels via Indirect Liquefaction, Ex Situ Catalytic Fast Pyrolysis, Hydrothermal Liquefaction, Combined Algal Processing, and Biochemical Conversion: Update of the 2019 State-of-Technology Cases. ANL/ESD20/2. Lemont, IL: Argonne National Laboratory. 


\section{Appendix A: 2014-2019 SOT and 2020-2022 Projection}

\section{Table A-1. Processing Area Cost Contributions and Key Technical Parameters}

\begin{tabular}{|c|c|c|c|c|c|c|c|c|c|c|c|c|}
\hline $\begin{array}{l}\text { Processing Area Cost Contributions \& Key Technical } \\
\text { Parameters }\end{array}$ & Units & 2014 SOT & 2015 SOT & 2016 Target & $2017 \mathrm{SOT}^{\Perp}$ & 2018 SOT & 2019 SOT & \begin{tabular}{|c|}
2020 \\
Projection \\
Option 1 \\
Co-HT \\
\end{tabular} & \begin{tabular}{|c|}
2020 \\
Projection \\
Option 2 \\
Yield $\uparrow$ \\
\end{tabular} & $\begin{array}{c}2021 \\
\text { Projection }\end{array}$ & $\begin{array}{l}2022 \\
\text { Projection }\end{array}$ & $\begin{array}{l}2030^{\neq+} \\
\text {Projection }\end{array}$ \\
\hline $\begin{array}{l}\text { Process Concept: Hydrocarbon Fuel Production } \\
\text { via Ex Situ Upgrading of Fast Pyrolysis Vapors }\end{array}$ & & Clean Pine & Clean Pine & Clean Pine & Clean Pine & Clean Pine & $\begin{array}{c}50 \% \text { Residues } \\
50 \% \text { Pine } \\
\end{array}$ & $\begin{array}{c}50 \% \text { Residues/ } \\
\text { 50\%Pine } \\
+\end{array}$ & $\begin{array}{c}50 \% \text { Residues } \\
50 \% \text { Pine }^{+t}\end{array}$ & $\begin{array}{c}75 \% \text { Residues } \mid \\
25 \% \text { Pine }\end{array}$ & \begin{tabular}{|c|}
$75 \%$ Residues \\
25\%Pine
\end{tabular} & \begin{tabular}{|c|}
$75 \%$ Residues/ \\
$25 \%$ Pine
\end{tabular} \\
\hline Year $\$$ Basis & & 2016 & 2016 & 2016 & 2016 & 2016 & 2016 & 2016 & 2016 & 2016 & 2016 & 2016 \\
\hline Projected Minimum Fuel Selling Price ${ }^{\boldsymbol{\Delta}}$ & $\$ / G^{\prime} E^{*}$ & $\$ 6.27$ & $\$ 5.44$ & $\$ 4.90$ & $\$ 4.09$ & $\$ 3.80$ & $\$ 3.33$ & $\$ 3.09$ & $\$ 3.09$ & $\$ 3.05$ & $\$ 3.00$ & $\$ 2.48$ \\
\hline Conversion Contribution & $\$ / G^{*}$ & $\$ 3.66$ & $\$ 3.30$ & $\$ 3.08$ & $\$ 2.82$ & $\$ 2.44$ & $\$ 2.14$ & $\$ 1.90$ & $\$ 2.02$ & $\$ 1.87$ & $\$ 1.83$ & $\$ 1.34$ \\
\hline Total Project Investment per Annual GGE & \$/GGE-yr & $\$ 18.50$ & $\$ 16.46$ & $\$ 14.94$ & $\$ 12.17$ & $\$ 12.47$ & $\$ 13.53$ & $\$ 12.32$ & $\$ 12.27$ & $\$ 12.19$ & $\$ 12.07$ & $\$ 11.13$ \\
\hline Plant Capacity (Dry Feedstock Basis) & metric tons/day & 2,000 & 2,000 & 2,000 & 2,000 & 2,000 & 2,000 & 2,000 & 2,000 & 2,000 & 2,000 & 2,000 \\
\hline Total Gasoline Equivalent Yield & GGE/dry US ton & 42 & 46 & 51 & 69 & 65 & 59 & 59 & 65 & 60 & 61 & 62 \\
\hline Diesel-Range Product Proportion (GGE* basis) & $\%$ of fuel product & $15 \%$ & $15 \%$ & $15 \%$ & $52 \%$ & $52 \%$ & $48 \%$ & $48 \%$ & $48 \%$ & $48 \%$ & $48 \%$ & $52 \%$ \\
\hline \multicolumn{13}{|l|}{ Feedstock } \\
\hline Total Cost Contribution" & $\$ / G G E$ & $\$ 2.60$ & $\$ 2.14$ & $\$ 1.82$ & $\$ 1.27$ & $\$ 1.36$ & $\$ 1.18$ & $\$ 1.19$ & $\$ 1.08$ & $\$ 1.18$ & $\$ 1.17$ & $\$ 1.14$ \\
\hline Capital Cost Contribution" & $\$ / G G E$ & $\$ 0.00$ & $\$ 0.00$ & $\$ 0.00$ & $\$ 0.00$ & $\$ 0.00$ & $\$ 0.00$ & $\$ 0.00$ & $\$ 0.00$ & $\$ 0.00$ & $\$ 0.00$ & $\$ 0.00$ \\
\hline Operating Cost Contribution" & $\$$ / GGE & $\$ 2.60$ & $\$ 2.14$ & $\$ 1.81$ & $\$ 1.27$ & $\$ 1.35$ & $\$ 1.18$ & $\$ 1.18$ & $\$ 1.07$ & $\$ 1.18$ & $\$ 1.16$ & $\$ 1.13$ \\
\hline Feedstock Cost" & $\$$ / Dry US Ton & $\$ 109.01$ & $\$ 98.31$ & $\$ 92.70$ & $\$ 87.82$ & $\$ 87.82$ & $\$ 70.15$ & $\$ 70.15$ & $\$ 70.15$ & $\$ 70.31$ & $\$ 70.31$ & $\$ 70.31$ \\
\hline Feedstock Moisture at Plant Gate & $\mathrm{wt} \% \mathrm{H}_{2} \mathrm{O}$ & $10 \%$ & $10 \%$ & $10 \%$ & $10 \%$ & $10 \%$ & $10 \%$ & $10 \%$ & $10 \%$ & $10 \%$ & $10 \%$ & $10 \%$ \\
\hline Feed Moisture Content to Pyrolyzer & wt $\% \mathrm{H}_{2} \mathrm{O}$ & $10 \%$ & $10 \%$ & $10 \%$ & $10 \%$ & $10 \%$ & $10 \%$ & $10 \%$ & $10 \%$ & $10 \%$ & $10 \%$ & $10 \%$ \\
\hline Energy Content (LHV, Dry Basis) & $\mathrm{BTU} / \mathrm{lb}$ & 8,000 & 8,000 & 8,000 & 8,000 & 8,000 & 7,900 & 7,900 & 7,900 & 8,000 & 8,000 & 8,000 \\
\hline \multicolumn{13}{|l|}{ Pyrolysis and Vapor Upgrading } \\
\hline Total Cost Contribution & $\$$ / GGE & $\$ 2.34$ & $\$ 2.03$ & $\$ 1.84$ & $\$ 1.46$ & $\$ 1.10$ & $\$ 1.14$ & $\$ 1.14$ & $\$ 1.06$ & $\$ 1.13$ & $\$ 1.12$ & $\$ 1.14$ \\
\hline Capital Cost Contribution & $\$$ / GGE & $\$ 0.95$ & $\$ 0.82$ & $\$ 0.74$ & $\$ 0.65$ & $\$ 0.60$ & $\$ 0.63$ & $\$ 0.63$ & $\$ 0.57$ & $\$ 0.62$ & $\$ 0.62$ & $\$ 0.63$ \\
\hline Operating Cost Contribution & $\$ / G G E$ & $\$ 1.39$ & $\$ 1.21$ & $\$ 1.09$ & $\$ 0.80$ & $\$ 0.50$ & $\$ 0.51$ & $\$ 0.51$ & $\$ 0.49$ & $\$ 0.50$ & $\$ 0.50$ & $\$ 0.51$ \\
\hline Ex Situ Reactor Configuration & reactor type & \begin{tabular}{|l|} 
Fluidized Bed \\
\end{tabular} & \begin{tabular}{|l|} 
Fluidized Bed \\
\end{tabular} & Fluidized Bed & Fixed Bed & Fixed Bed & Fixed Bed & Fixed Bed & Fixed Bed & Fixed Bed & Fixed Bed & Fixed Bed \\
\hline Ratio of Online:Regenerating Fixed Bed Reactors & ratio & $\mathrm{N} / \mathrm{A}$ & $\mathrm{N} / \mathrm{A}$ & $\mathrm{N} / \mathrm{A}$ & 2:5 & $2: 3$ & $2: 2$ & $2: 2$ & $2: 2$ & $2: 2$ & $2: 2$ & $2: 2$ \\
\hline Gas Phase & wt $\%$ of dry biomass & $35 \%$ & $36 \%$ & $34 \%$ & $31 \%$ & $35 \%$ & $38 \%$ & $38 \%$ & $34 \%$ & $38 \%$ & $38 \%$ & $31 \%$ \\
\hline Aqueous Phase & wt $\%$ of dry biomass & $25 \%$ & $25 \%$ & $24 \%$ & $27 \%$ & $22 \%$ & $24 \%$ & $24 \%$ & $26 \%$ & $24 \%$ & $24 \%$ & $23 \%$ \\
\hline Carbon Loss & $\%$ of $\mathrm{C}$ in biomass & $2.9 \%$ & $2.9 \%$ & $3.4 \%$ & $2.9 \%$ & $5.0 \%$ & $4.4 \%$ & $4.4 \%$ & $4.3 \%$ & $4.4 \%$ & $4.4 \%$ & $3.0 \%$ \\
\hline Organic Phase & wt $\%$ of dry biomass & $17.5 \%$ & $18.6 \%$ & $21.8 \%$ & $28.3 \%$ & $27.9 \%$ & $23.2 \%$ & $23.2 \%$ & $25.6 \%$ & $23.4 \%$ & $23.4 \%$ & $31.4 \%$ \\
\hline H/C Molar Ratio & ratio & 1.1 & 1.1 & 1.1 & 1.2 & 1.2 & 1.2 & 1.2 & 1.2 & 1.2 & 1.2 & 1.2 \\
\hline Oxygen & wt $\%$ of organic phase & $15.0 \%$ & $13.3 \%$ & $16.8 \%$ & $16.5 \%$ & $18.6 \%$ & $15.1 \%$ & $15.1 \%$ & $15.2 \%$ & $15.1 \%$ & $15.1 \%$ & $16.4 \%$ \\
\hline Carbon Efficiency & $\%$ of $\mathrm{C}$ in biomass & $27 \%$ & $29 \%$ & $33 \%$ & $42 \%$ & $40 \%$ & $35 \%$ & $35 \%$ & $39 \%$ & $35 \%$ & $35 \%$ & $47 \%$ \\
\hline Solid Losses (Char + Coke) & wt $\%$ of dry biomass & $23 \%$ & $21 \%$ & $20 \%$ & $14 \%$ & $15 \%$ & $14 \%$ & $14 \%$ & $14 \%$ & $14 \%$ & $14 \%$ & $15 \%$ \\
\hline Char & wt $\%$ of dry biomass & $12.0 \%$ & $11.0 \%$ & $12.0 \%$ & $10.4 \%$ & $11.7 \%$ & $11.6 \%$ & $11.6 \%$ & $11.6 \%$ & $11.7 \%$ & $11.7 \%$ & $11.7 \%$ \\
\hline Coke & wt $\%$ of dry biomass & $11.0 \%$ & $9.5 \%$ & $8.3 \%$ & $3.3 \%$ & $3.7 \%$ & $2.3 \%$ & $2.3 \%$ & $2.2 \%$ & $2.3 \%$ & $2.3 \%$ & $3.2 \%$ \\
\hline \multicolumn{13}{|l|}{ Vapor Quench, Co-Product Recovery + Contingency } \\
\hline Total Cost Contribution & $\$ / G G E$ & $\$ 0.35$ & $\$ 0.33$ & $\$ 0.28$ & $\$ 0.20$ & $\$ 0.22$ & $\$ 0.34$ & $\$ 0.42$ & $\$ 0.30$ & $\$ 0.40$ & $\$ 0.40$ & $\$ 0.23$ \\
\hline Capital Cost Contribution & $\$$ / GGE & $\$ 0.20$ & $\$ 0.19$ & $\$ 0.16$ & $\$ 0.12$ & $\$ 0.13$ & $\$ 0.22$ & $\$ 0.26$ & $\$ 0.19$ & $\$ 0.24$ & $\$ 0.24$ & $\$ 0.13$ \\
\hline Operating Cost Contribution & $\$ / G G E$ & $\$ 0.15$ & $\$ 0.14$ & $\$ 0.12$ & $\$ 0.08$ & $\$ 0.09$ & $\$ 0.12$ & $\$ 0.16$ & $\$ 0.11$ & $\$ 0.16$ & $\$ 0.16$ & $\$ 0.10$ \\
\hline
\end{tabular}

(continued next page) 
(continued from previous page)

\begin{tabular}{|c|c|c|c|c|c|c|c|c|c|c|c|c|}
\hline \multicolumn{13}{|l|}{ Hydroprocessing \& Separation / Refinery Co-Processing } \\
\hline Total Cost Contribution & $\$ / G G E$ & $\$ 0.33$ & $\$ 0.31$ & $\$ 0.34$ & $\$ 0.35$ & $\$ 0.38$ & $\$ 0.30$ & $\$ 0.21$ & $\$ 0.30$ & $\$ 0.21$ & $\$ 0.21$ & $\$ 0.04$ \\
\hline Capital Cost Contribution & $\$ / G G E$ & $\$ 0.17$ & $\$ 0.16$ & $\$ 0.18$ & $\$ 0.19$ & $\$ 0.20$ & $\$ 0.16$ & $\$ 0.00$ & $\$ 0.16$ & $\$ 0.00$ & $\$ 0.00$ & $\$ 0.00$ \\
\hline Operating Cost Contribution & $\$ /$ GGE & $\$ 0.15$ & $\$ 0.14$ & $\$ 0.16$ & $\$ 0.16$ & $\$ 0.18$ & $\$ 0.14$ & $\$ 0.21$ & $\$ 0.14$ & $\$ 0.21$ & $\$ 0.21$ & $\$ 0.04$ \\
\hline Carbon Efficiency of Organic Liquid Feed to Fuels & $\%$ & $88.4 \%$ & $89.5 \%$ & $87.2 \%$ & $91.0 \%$ & $89.0 \%$ & $93.5 \%$ & $93.5 \%$ & $93.5 \%$ & $93.5 \%$ & $93.5 \%$ & $91.0 \%$ \\
\hline Hydrotreating Pressure & psia & 2,000 & 2,000 & 2,000 & 1,900 & 1,900 & 1,900 & 1,900 & 1,900 & 1,900 & 1,900 & 1,900 \\
\hline Oxygen Content in Cumulative Fuel Product & wt \% & $0.8 \%$ & $0.8 \%$ & $0.8 \%$ & $0.6 \%$ & $0.5 \%$ & $0.5 \%$ & $0.5 \%$ & $0.5 \%$ & $0.5 \%$ & $0.5 \%$ & $0.6 \%$ \\
\hline \multicolumn{13}{|l|}{ Hydrogen Production } \\
\hline Total Cost Contribution & $\$ / G G E$ & $\$ 0.61$ & $\$ 0.56$ & $\$ 0.60$ & $\$ 0.62$ & $\$ 0.51$ & $\$ 0.61$ & $\$ 0.44$ & $\$ 0.60$ & $\$ 0.44$ & $\$ 0.44$ & $\$ 0.46$ \\
\hline Capital Cost Contribution & $\$ / G G E$ & $\$ 0.39$ & $\$ 0.36$ & $\$ 0.38$ & $\$ 0.41$ & $\$ 0.33$ & $\$ 0.39$ & $\$ 0.28$ & $\$ 0.37$ & $\$ 0.28$ & $\$ 0.28$ & $\$ 0.28$ \\
\hline Operating Cost Contribution & $\$ / G G E$ & $\$ 0.22$ & $\$ 0.20$ & $\$ 0.22$ & $\$ 0.21$ & $\$ 0.18$ & $\$ 0.22$ & $\$ 0.16$ & $\$ 0.24$ & $\$ 0.16$ & $\$ 0.16$ & $\$ 0.17$ \\
\hline Additional Natural Gas (NG) at the Biorefinery & $\%$ of biomass LHV & $0.3 \%$ & $0.1 \%$ & $0.2 \%$ & $0.1 \%$ & $0.3 \%$ & $0.1 \%$ & $0.5 \%$ & $1.3 \%$ & $0.6 \%$ & $0.6 \%$ & $0.2 \%$ \\
\hline \multicolumn{13}{|l|}{ CoProducts } \\
\hline Total Cost Contribution & $\$ / G G E$ & & & & & & $(\$ 0.52)$ & $(\$ 0.52)$ & $(\$ 0.47)$ & $(\$ 0.52)$ & $(\$ 0.53)$ & $(\$ 0.74)$ \\
\hline Capital Cost Contribution ${ }^{\bullet}$ & $\$ / G G E$ & & & & & & & & & & & $\$ 0.06$ \\
\hline Operating Cost Contribution ${ }^{\circ}$ & $\$ / G G E$ & & & & & & & & & & & $(\$ 0.81)$ \\
\hline CoProduct Credit & $\$ / G G E^{*}$ & & & & & & $(\$ 0.52)$ & $(\$ 0.52)$ & $(\$ 0.47)$ & $(\$ 0.52)$ & $(\$ 0.53)$ & $(\$ 0.83)$ \\
\hline \multicolumn{13}{|l|}{ Balance of Plant } \\
\hline Total Cost Contribution & $\$ / G G E$ & $\$ 0.04$ & $\$ 0.07$ & $\$ 0.03$ & $\$ 0.20$ & $\$ 0.23$ & $\$ 0.27$ & $\$ 0.20$ & $\$ 0.21$ & $\$ 0.20$ & $\$ 0.19$ & $\$ 0.22$ \\
\hline Capital Cost Contribution & $\$ / G G E$ & $\$ 0.80$ & $\$ 0.71$ & $\$ 0.56$ & $\$ 0.43$ & $\$ 0.46$ & $\$ 0.45$ & $\$ 0.52$ & $\$ 0.38$ & $\$ 0.52$ & $\$ 0.51$ & $\$ 0.41$ \\
\hline Operating Cost Contribution & $\$ /$ GGE & $(\$ 0.76)$ & $(\$ 0.64)$ & $(\$ 0.54)$ & $(\$ 0.23)$ & $(\$ 0.23)$ & $(\$ 0.18)$ & $(\$ 0.32)$ & $(\$ 0.17)$ & $(\$ 0.32)$ & $(\$ 0.32)$ & $(\$ 0.20)$ \\
\hline $\begin{array}{l}\text { Electricity Production from Steam Turbine (credit included in op. } \\
\text { cost above) }\end{array}$ & $\$ / G E^{*}$ & $(\$ 1.12)$ & $(\$ 0.96)$ & $(\$ 0.78)$ & $(\$ 0.42)$ & $(\$ 0.45)$ & $(\$ 0.40)$ & $(\$ 0.57)$ & $(\$ 0.37)$ & $(\$ 0.57)$ & $(\$ 0.57)$ & $(\$ 0.41)$ \\
\hline \multicolumn{13}{|l|}{ Sustainability and Process Efficiency Metrics } \\
\hline Fuel and Coproducts Yield by Weight of Biomass & $\% \mathrm{w} / \mathrm{w}$ of dry biomass & $13.7 \%$ & $15.0 \%$ & $16.5 \%$ & $22.2 \%$ & $20.9 \%$ & $22.5 \%$ & $22.5 \%$ & $24.4 \%$ & $22.7 \%$ & $22.8 \%$ & $24.8 \%$ \\
\hline Carbon Efficiency of Biomass to Fuels and Coproducts & $\%$ C in Feedstock & $23.5 \%$ & $25.9 \%$ & $28.3 \%$ & $38.1 \%$ & $35.9 \%$ & $37.2 \%$ & $37.2 \%$ & $40.5 \%$ & $37.2 \%$ & $37.3 \%$ & $42.4 \%$ \\
\hline Overall Carbon Efficiency to Liquid Hydrocarbon Fuels & $\%$ C in Feedstock & $23.5 \%$ & $25.9 \%$ & $28.3 \%$ & $38.1 \%$ & $35.9 \%$ & $33.0 \%$ & $33.0 \%$ & $36.3 \%$ & $33.0 \%$ & $33.0 \%$ & $33.9 \%$ \\
\hline Overall Energy Efficiency to Liquid Hydrocarbon Fuels & $\%$ LHV of Feedstock & $30.5 \%$ & $33.4 \%$ & $37.1 \%$ & $50.3 \%$ & $47.2 \%$ & $43.6 \%$ & $43.6 \%$ & $48.0 \%$ & $43.6 \%$ & $43.6 \%$ & $44.9 \%$ \\
\hline Electricity Production & kWh/GGE & 21.0 & 18.0 & 14.7 & 8.0 & 8.7 & 7.8 & 10.8 & 6.3 & 10.8 & 10.7 & 7.9 \\
\hline Electricity Consumption (Entire Process) & kWh/GGE & 12.7 & 11.0 & 9.6 & 6.4 & 7.5 & 7.4 & 7.1 & 6.7 & 7.1 & 7.0 & 7.4 \\
\hline Water Consumption in Conversion Process & gal $\mathrm{H}_{2} \mathrm{O} / \mathrm{GGE}$ & 1.4 & 1.4 & 1.3 & 1.5 & 1.4 & 1.7 & 1.2 & 1.5 & 1.2 & 1.2 & 1.4 \\
\hline TEA Reference File & & \begin{tabular}{|c|} 
PyVPU-V218g \\
ES - Fluidized \\
Bed - FY14 \\
SOT (2016\$)- \\
Vo2d-feed- \\
109.01.xlsm
\end{tabular} & $\begin{array}{c}\text { PyVPU-V18g } \\
\text { ES - Fluidized } \\
\text { Bed - FY15 } \\
\text { SOT (2016\$)- } \\
\text { Vo2d-feed- } \\
\text { 98.31.xlsm }\end{array}$ & \begin{tabular}{|c} 
PyVPU-V18g \\
ES - Fluidized \\
Bed - FY16 \\
SOT (2016\$)- \\
Vo2d-feed- \\
92.70.xlsm
\end{tabular} & \begin{tabular}{|c} 
PyVPU-V218h \\
ES FixedBed- \\
v49-r046-AP10- \\
FY17SOT \\
(2016\$)-V02d- \\
b.xlsm
\end{tabular} & $\begin{array}{l}\text { PyVPU-V18h } \\
\text { ES FixedBed- } \\
\text { v49-r046-AP10- } \\
\text { FY18SOT } \\
\text { (2016\$)-Vo9d- } \\
\text { Revised } \\
\text { 40.4pct C-Eff- } \\
\text { b.xlsm }\end{array}$ & $\begin{array}{c}\text { PyVPU-V218h } \\
\text { ES FixedBed- } \\
\text { v49-r046-AP 10- } \\
\text { FY19SOT-V18- } 18 \text { - } \\
\text { Acetone-MEK- } \\
\text { \$3.33.xIsm }\end{array}$ & $\begin{array}{c}\text { PyVPU-V18h } \\
\text { ES FixedBed- } \\
\text { v49-r046-AP10- } \\
\text { FY20Target- } \\
\text { V18-Acetone- } \\
\text { MEK- } \\
\text { HTcoproc-03b- } \\
\text { \$3.09.xlsm }\end{array}$ & \begin{tabular}{|c|} 
PyVPU-V218h \\
ES FixedBed- \\
v49-r046-AP10- \\
FY20Target- \\
V18-Acetone- \\
MEK-3pct- \\
higher-C-eff-03- \\
$\$ 3.09 . x \mid s m$ \\
\end{tabular} & $\begin{array}{c}\text { PyVPU-V218h } \\
\text { ES FixedBed- } \\
\text { v99-1046-AP 10- } \\
\text { FY21 Target- } \\
\text { V18-Acetone- } \\
\text { MEK- } \\
\text { HTcoproc-03b- } \\
\text { Low Ash- } \\
\text { \$3.05.xlsm }\end{array}$ & $\begin{array}{c}\text { PyVPU--218h } \\
\text { ES FixedBed- } \\
\text { va9-ro46-AP 10 } \\
\text { FY22Target- } \\
\text { V18-Acetone- } \\
\text { MEK- } \\
\text { HTcoproc-03b- } \\
\text { Low Ash- } \\
\text { \$3.00.xlsm }\end{array}$ & 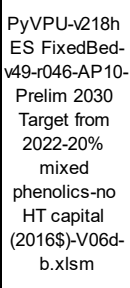 \\
\hline
\end{tabular}

CConceptual design result. †SOT: State of Technology. *Gallon Gasoline Equivalent (GGE) on a Lower Heating Value (LHV) basis. ** Natural gas stream was negligible in most of the biorefinery models. This was included to maintain model flexibility to allow natural gas use as an option. ${ }^{\ddagger \ddagger} 2030$ projections are based on high-level estimates and will be modeled in detail in future years. It is proposed that co-

hydroprocessing of CFP oil will occur at a petroleum refinery. Capital for hydrogen production is included, while natural gas feed for hydrogen production is not included because credit is not taken for an equivalent ano than $1 \%$ for all other years. "1" An additional biomass heater is included as a small additional in-plant cost, as shown in https://www.nrel.gov/docs/fy 150 osti/62455. pdf. ${ }^{\lrcorner 1}$ For the 2017 SOT unquantified portion of CFP yields were prorated to solids, liquids, and gases using measured yields. "1 Small adjustments made to previously published feedstock cost estimates for $2014-2016$. 